\title{
Microbial Metabolism of Theaflavin-3,3'-digallate and Its Gut Microbiota Composition Modulatory Effects
}

\author{
Zhibin Liu, Wouter J. C. de Bruijn, Marieke E. Bruins, and Jean-Paul Vincken*
}

Cite This: J. Agric. Food Chem. 2021, 69, 232-245

Read Online

ABSTRACT: Theaflavin-3,3'-digallate (TFDG), a bioactive black tea phenolic, is poorly absorbed in the small intestine, and it has been suggested that gut microbiota metabolism plays a crucial role in its bioactivities. However, information on its metabolic fate and impact on gut microbiota is limited. Here, TFDG was anaerobically fermented in vitro by human fecal microbiota, and epigallocatechin gallate (EGCG) was used for comparison. Despite the similar flavan-3-ol skeletons, TFDG was more slowly degraded and yielded a distinctively different metabolic profile. The formation of theanaphthoquinone as the main metabolites was unique to TFDG. Additionally, a number of hydroxylated phenylcarboxylic acids were formed with low concentrations, when comparing to EGCG metabolism. Microbiome profiling demonstrated several similarities in gut microbiota modulatory effects, including growth-promoting effects on Bacteroides, Faecalibacterium, Parabacteroides, and Bifidobacterium, and inhibitory effects on Prevotella and Fusobacterium. In conclusion, TFDG and EGCG underwent significantly different microbial metabolic fates, yet their gut microbiota modulatory effects were similar.

KEYWORDS: $16 S$ rRNA sequencing, colonic fermentation, microbial metabolic fate, theaflavins, UHPLC-Q-Orbitrap-MS

\section{INTRODUCTION}

Theaflavins are one of the major characteristic phenolic compounds contributing to the color, taste, and beneficial health effects of black tea. ${ }^{1}$ Four well-characterized theaflavins were documented to exist in black tea, including theaflavin (TF), theaflavin-3-gallate (TF3G), theaflavin-3'-gallate (TF3'G), and theaflavin-3,3'-digallate (TFDG). Theaflavins are characterized by their $1^{\prime}, 2^{\prime}$-dihydroxy-3,4-benzotropolone moiety (Figure 1). ${ }^{2}$ This group of phenolic compounds are<smiles>[R20]OC1Cc2c(O)cc(O)cc2OC1c1cc([R20])c(O)c(O)c1</smiles><smiles></smiles>

$\begin{array}{llllll} & \mathbf{R}_{\mathbf{1}} & \mathbf{R}_{\mathbf{2}} & & \mathbf{R}_{\mathbf{3}} & \mathbf{R}_{\mathbf{4}} \\ \text { EC } & \text { H } & \text { H } & \text { TF } & \text { H } & \text { H } \\ \text { EGC } & \text { OH } & \text { H } & \text { TF3G } & \text { G } & \text { H } \\ \text { ECG } & \text { H } & \text { G } & \text { TF3'G } & \text { H } & \text { G } \\ \text { EGCG } & \text { OH } & \text { G } & \text { TFDG } & \text { G } & \text { G }\end{array}$

Figure 1. Chemical structures of major catechins and theaflavins in green tea and black tea. Blue shading in the theaflavin structure highlights the characteristic $1^{\prime}, 2^{\prime}$-dihydroxy-3,4-benzotropolone moiety. products of the oxidation and dimerization of catechins, the major phenolic compounds in green tea, by endogenous polyphenol oxidases and peroxidases during the fermentation process of black tea., ${ }^{3,4}$ Of the four theaflavins, TFDG was reported as the most abundant one in black tea. ${ }^{5}$ TFDG is formed by the condensation of epicatechin gallate (ECG) and epigallocatechin gallate (EGCG) via the fusion of their respective catechol-type and pyrogallol-type B-rings. ${ }^{4}$

Phenolic compounds, including catechins and theaflavins, have been reported to possess numerous health benefits; however, it is also well known that many of them are poorly absorbed in the small intestine. ${ }^{6}$ Approximately $70 \%$ of the ingested monomeric catechins were reported to be recovered in the large intestine. ${ }^{7}$ Dimeric derivatives of catechins, such as theaflavins, have even lower bioavailability in the small intestine. It is reported that theaflavins and their phase II metabolites were not detected in urine excreted $0-30 \mathrm{~h}$ after intake. $^{8}$ Therefore, a substantial proportion of consumed catechins and theaflavins will enter the large intestine, where they can be subject to bioconversion by resident microorganisms. A better understanding of the colonic metabolic fate of tea and its components is essential for the interpretation of their health-promoting effects.

The metabolic fate of green tea catechins in the colon has been studied extensively. ${ }^{9-12}$ The general consensus in the

Received: October 18, 2020

Revised: November 30, 2020

Accepted: December 9, 2020

Published: December 21, 2020

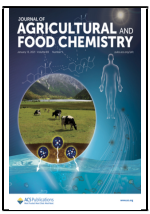


field is that catechins are bioconverted into a series of (hydroxylated) phenylcarboxylic acids through consecutive ester hydrolysis, C-ring opening, A-ring fission, dehydroxylation, and aliphatic chain shortening. ${ }^{11,13,14}$ On the other hand, the metabolic fate of theaflavins and other black tea phenolics has been studied to a lesser extent, despite the fact that black tea is more widely consumed than green tea. ${ }^{2}$ To date, only three papers have been published regarding the microbial metabolism of theaflavins. It was reported that TFDG could be degraded to TF, TF3G, and TF3' G by gut microbiota. ${ }^{15,16}$ Following the degalloylation, theaflavins could be further converted to some smaller phenolic compounds, such as 5-( $3^{\prime}, 4^{\prime}$-dihydroxyphenyl)- $\gamma$-valerolactone and 3- $\left(3^{\prime}, 4^{\prime}\right.$ dihydroxyphenyl)propionic acid. ${ }^{8}$ Similar metabolites were also identified upon microbial metabolism of green tea catechins. ${ }^{11}$ Considering that theaflavins are formed by the condensation of green tea catechins, similar metabolites can be expected from microbial metabolism of catechins and theaflavins. However, critical knowledge gaps still exist about the rate of theaflavin metabolism, the abundance of individual metabolites, and the possibility that unique metabolites may be formed. The comparison of the colonic metabolic fates of pure TFDG and EGCG will help in filling these knowledge gaps.

Besides the formation of metabolites by gut microbiota, there is evidence from in vitro and in vivo studies, which shows that pure catechins and green or black tea extracts could alter the composition and metabolic activities of gut microbiota toward a healthier profile. ${ }^{17-19}$ It was reported that consumption of green or black tea with a standardized phenolic content results in similar gut microbiota modulatory effects, including the growth-promoting effects on Lachnospiraceae and Akkermansia, and inhibitory effects on Clostridium leptum. ${ }^{20}$ Nonetheless, the effects of dimerization of catechins on gut microbiota modulation are poorly understood and should be further investigated using purified compounds. To the best of our knowledge, this is the first study that reports on the gut microbiota modulatory effects of pure galloylated theaflavin, and how it compares to green tea catechins in this respect.

Theaflavins and green tea catechins share similar flavan-3-ol building blocks. It is therefore expected that the fermentation of these compounds will result in the formation of similar metabolites, i.e., mainly (hydroxylated) phenylcarboxylic acids, and that they will exhibit similar gut microbiota modulatory effects. We aim to perform a direct comparison between TFDG and EGCG with respect to their microbial metabolism and gut microbiota composition modulatory effects in an in vitro anaerobic fermentation.

\section{MATERIALS AND METHODS}

Chemicals. Theaflavin-3,3'-digallate (TFDG) and theaflavin (TF), both with purity of $98 \%$, were purchased from Chromadex (Santa Ana, CA, USA). Epicatechin (EC), epigallocatechin (EGC), epicatechin gallate (ECG), epigallocatechin gallate (EGCG), gallic acid, pyrogallol, 4-hydroxybenzoic acid, 4-hydroxyphenylacetic acid, 4-phenylbutyric acid, 5-(4'-hydroxyphenyl)valeric acid, all with purities of at least 98\%, were purchased from Sigma Aldrich (St. Louis, MO, USA). Ultra-high performance liquid chromatography/ mass spectrometry (UHPLC-MS) grade acetonitrile (ACN), ACN with $0.1 \%(\mathrm{v} / \mathrm{v})$ formic acid, and water with $0.1 \%(\mathrm{v} / \mathrm{v})$ formic acid were purchased from Biosolve (Valkenswaard, The Netherlands). Water for other purposes than UHPLC-MS was prepared using a Milli-Q water purification system (Millipore, Billerica, MA, USA).
In Vitro Fermentation of TFDG and EGCG with Human Gut Microbiota. The in vitro fecal fermentation of TFDG and EGCG was performed following the methodology described by Gu et al. ${ }^{21}$ with some modifications. Fecal materials were obtained from four healthy volunteers (three males and one female, 24-38 years old), who reported no consumption of tea in the week prior to the donation and declared no antibiotic treatment in 3 months prior to the donation. After collection, all the fecal materials were stored at $-80{ }^{\circ} \mathrm{C}$. Equal amounts of fecal materials $(1.0 \mathrm{~g})$ from the four volunteers were transferred to an anaerobic chamber $\left(10 \% \mathrm{H}_{2}, 5 \% \mathrm{CO}_{2}\right.$, and $85 \% \mathrm{~N}_{2}$; Bactron, Cornelius, OR, USA) to thaw for $3 \mathrm{~h}$ at $37^{\circ} \mathrm{C}$, and after thawing, they were mixed with culture medium in a ratio of $1: 40(\mathrm{w} /$ v). The culture medium was a phenol-free standard ileal efflux medium (SIEM), which simulates the fermentation conditions of food components in the human colon. ${ }^{22}$ All ingredients were purchased from Tritium Microbiologie (Veldhoven, The Netherlands). After mixing fecal material and culture medium, the fecal slurries of the four volunteers were pooled and homogenized and then further strained through four layers of cheese cloth to obtain a homogeneous human fecal suspension (HFS). This HFS was incubated at $37{ }^{\circ} \mathrm{C}$ in the anaerobic chamber for $12 \mathrm{~h}$ to activate the bacteria. Subsequently, aliquots of $4.5 \mathrm{~mL}$ of HFS were spiked with a $0.5 \mathrm{~mL}$ TFDG solution $(0.5 \mathrm{mmol} / \mathrm{L})$, EGCG solution $(1 \mathrm{mmol} / \mathrm{L})$, or water. The final concentrations of TFDG and EGCG were 50 and $100 \mu \mathrm{mol} / \mathrm{L}$, respectively. The mixtures were then incubated at $37{ }^{\circ} \mathrm{C}$ in the anaerobic chamber for $48 \mathrm{~h}$. All fermentations were performed in quadruplicate. For the analysis of TFDG, EGCG, and their metabolites, $100 \mu \mathrm{L}$ samples were taken after $0,2,6,12,24,36$, and $48 \mathrm{~h}$ of fermentation and immediately diluted in $300 \mu \mathrm{L}$ of ACN to stop the fermentation. After centrifugation $\left(30 \mathrm{~min}, 22,000 \mathrm{~g}, 4^{\circ} \mathrm{C}\right)$, the supernatants were collected and stored at $-20{ }^{\circ} \mathrm{C}$ until UHPLCHRMS analysis. For gut microbiota analysis, $1 \mathrm{~mL}$ of the samples at fermentation times of $0,12,24$, and $48 \mathrm{~h}$ were collected and immediately frozen at $-80{ }^{\circ} \mathrm{C}$ until bacterial DNA extraction.

Analysis of the Microbial Metabolites of TFDG and EGCG by UHPLC-HRMS. Chromatographic separations were performed on a Vanquish UHPLC system (Thermo Fisher Scientific, Bremen, Germany) equipped with a binary pump, split loop autosampler, column compartment, and diode array detector. Samples were separated on an Acquity UHPLC BEH C18 column (150 mm $\times$ $2.1 \mathrm{~mm}, 1.7 \mu \mathrm{m}$; Waters, Milford, MA) with a VanGuard guard column of the same material $(5 \mathrm{~mm} \times 2.1 \mathrm{~mm}, 1.7 \mu \mathrm{m}$; Waters, Milford, MA). The column compartment heater was operated in still air mode at $45{ }^{\circ} \mathrm{C}$, and the post-column cooler was set to $40{ }^{\circ} \mathrm{C}$. The injection volume was $1.0 \mu \mathrm{L}$. Mobile phases consisting of $0.1 \%(\mathrm{v} / \mathrm{v})$ formic acid in water (A) and $0.1 \%(\mathrm{v} / \mathrm{v})$ formic acid in ACN (B) were used at a flow rate of $400 \mu \mathrm{L} / \mathrm{min}$. The elution program was set as follows: isocratic at $1 \%(\mathrm{v} / \mathrm{v})$ B for $2 \mathrm{~min} ; 2-22 \mathrm{~min}$ linear gradient to $99 \%(\mathrm{v} / \mathrm{v}) \mathrm{B} ; 22-25 \mathrm{~min}$ isocratic at $99 \%(\mathrm{v} / \mathrm{v}) \mathrm{B}$. The mobile phase was adjusted to starting conditions in $1 \mathrm{~min}$ followed by equilibration for $4 \mathrm{~min}$.

For mass spectrometric analysis, a Thermo Q-Exactive hybrid quadrupole-Orbitrap mass spectrometer (Thermo Fisher Scientific, Bremen, Germany) equipped with a heated ESI source was connected in line to the UHPLC system. The mass spectrometer was operated in both negative and positive mode. The ESI parameters were set as follows: spray voltage, $+3500 \mathrm{~V} /-3000 \mathrm{~V}$; atomization temperature, $350{ }^{\circ} \mathrm{C}$; sheath gas (nitrogen) pressure, 50 arb; auxiliary gas (nitrogen) pressure, $12.5 \mathrm{arb}$; capillary temperature, $350{ }^{\circ} \mathrm{C}$; S-lens $\mathrm{RF}, 50 \mathrm{~V}$; resolution, MS full scan 70,000 full width at half maximum (FWHM), MS/MS 17,500 FWHM; scan range, $m / z$ 100-1500; scanning mode, full scan to data-dependent MS/MS (intensity threshold 800,000). An external calibration for mass accuracy was performed before the analysis according to the manufacturer's guidelines. Instrument control and data acquisition were performed with Xcalibur software (version 4.1, Thermo Fisher Scientific, Bremen, Germany). External standards of TFDG, TF, EGCG, ECG, EGC, EC, gallic acid, pyrogallol, 4-hydroxybenzoic acid, 4hydroxyphenylacetic acid, 4-phenylbutyric acid, and 5-(4'hydroxyphenyl)valeric acid were used for qualification and 
Figure 2.

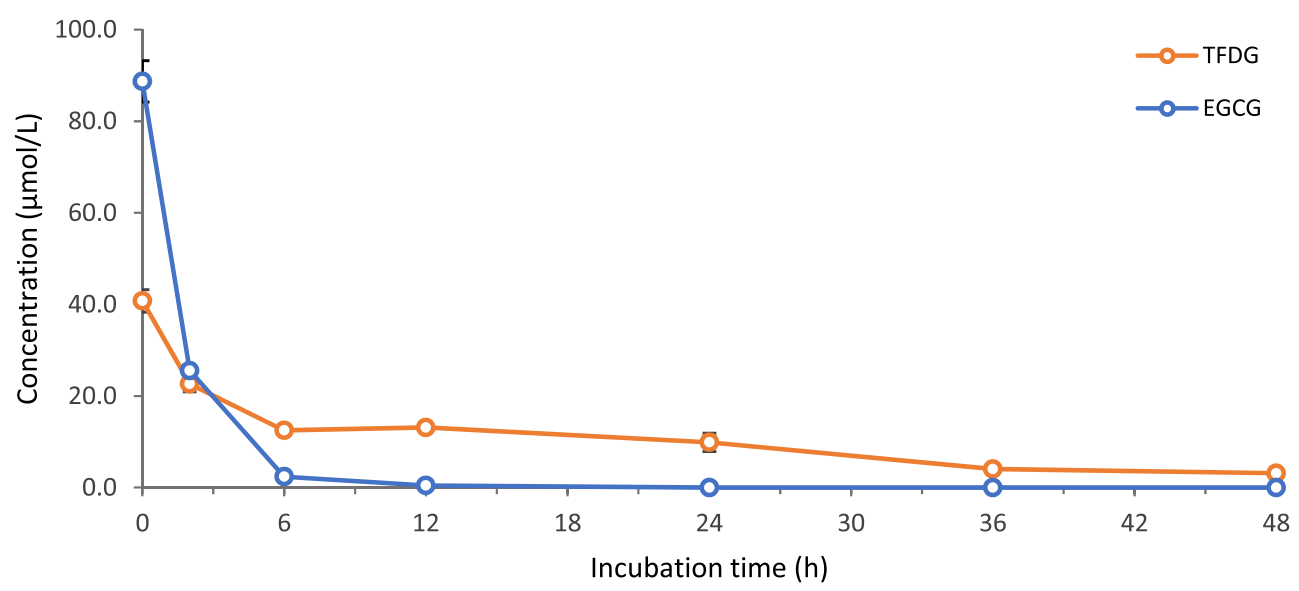

Figure 2. Changes in the concentration of TFDG and EGCG throughout the fermentation.

quantification of the metabolites detected by comparing retention times and accurate masses. The concentrations of these compounds and compounds with similar structures were further calculated based on the respective calibration curves $\left(0.1-100 \mu \mathrm{mol} / \mathrm{L}, R^{2}>0.99\right)$. In addition, a quality control sample containing $10 \mu \mathrm{mol} / \mathrm{L}$ EGCG was injected to UHPLC-HRMS every 12 samples to assess system stability. The relative standard deviation of peak intensities was approximately $20 \%$, and the retention time shifts was lower than 0.3 min, which indicated that the analytic platform was stable and reliable.

In order to investigate the effect of TFDG and EGCG to the general metabolic profiles after $48 \mathrm{~h}$ of fermentation, an untargeted metabolomics approach was applied. The raw LC-MS data files acquired at $48 \mathrm{~h}$ were processed by Compound Discoverer (version 3.1, Thermo Fisher Scientific, Bremen, Germany) with an untargeted metabolomics workflow (Figure S1, Supporting Information). This workflow includes retention time alignment, unknown compound detection, compound grouping, and gaps filling across all samples. Briefly, after data input, peak alignment was performed using a linear model with $5 \mathrm{ppm}$ mass accuracy tolerances and 0.5 min retention time shift. Subsequently, compounds were detected based on the following criteria: mass accuracy tolerances, $5 \mathrm{ppm}$; retention time tolerances, $0.5 \mathrm{~min}$; intensity tolerance for isotope search, $30 \%$; minimum peak intensity, $10^{5}$; signal-to-noise ratio threshold, 3; maximum peak width for detection, $0.5 \mathrm{~min}$. The detected unknown compounds were then grouped among all raw data files. Afterward, the missing peaks were automatically filled with chromatographic peaks based on the spectrum noise level. The peaks were then tentatively identified by matching the mzCloud and ChemSpider databases with accurate mass, isotopic pattern, and fragment ions.

The detected metabolites together with their peak intensities were used for further multivariate data analysis. First, the overall differences among all samples were evaluated through the principal coordinates analysis (PCoA), by using the ade4 package in R (Version 3.6.1). Subsequently, the partial least-squares discriminant analysis (PLSDA) was applied to further compare the different treatment groups, by using the DiscriMiner package in $\mathrm{R}$. In addition, variable importance for the projection (VIP) from PLS-DA modeling was used to identify the metabolites for distinguishing the different treatments.

Analysis of Gut Microbiota Composition after Fermentation with TFDG and EGCG. Genomic DNA was extracted from each sample by using the QIAamp Fast DNA Stool Mini Kit (Qiagen, Germany), according to the manufacturer's instructions. The quantity and quality of the obtained DNA were then checked by $1 \%$ agarose gel electrophoresis and stored at $-20^{\circ} \mathrm{C}$ until use. The $\mathrm{V} 3-\mathrm{V} 4$ region of bacterial $16 \mathrm{~S}$ rRNA genes of the gut microbiota strains was amplified by employing two universal bacterial primers, 341-F (5'CCT AYG GGR BGC ASC AG-3') and 806-R (5'- GGA CTA CNN
GGG TAT CTA AT-3') with specific barcodes. After amplification, PCR products were checked by $1 \%$ agarose gel electrophoresis. Samples with bright main strip between 450 and 550 bp were selected and purified with the QIAquick Gel Extraction Kit (Qiagen, Germany). Subsequently, the sequencing library of bacterial $16 \mathrm{~S}$ rRNA genes was generated by utilizing the NEBNext Ultra IIDNA Library Prep Kit (NEB, USA), and DNA samples were paired-end sequenced on an Illumina NovaSeq 6000 platform.

Raw sequencing reads obtained from the Illumina platform were then merged using FLASH (version 1.2.7), ${ }^{23}$ and filtered with the QIIME (version 1.7). ${ }^{24}$ All quality filtered sequencing reads were then clustered into operational taxonomic units (OTUs) with $97 \%$ sequence similarity, by using Uparse (version 7.0). ${ }^{25}$ The representative sequence for each bacterial OTU was then annotated against the Silva SSU rRNA database with Mothur (version 1.30.2). ${ }^{26}$ The relative abundance of each OTU across all samples was calculated and used for further data mining. A hierarchical clustering dendrogram was constructed with Ward's method to determine the similarity across all samples, by using the factoextra package in $\mathrm{R}$. The overall differences among all samples were evaluated through principal component analysis (PCA), by using the ade4 package in R. Subsequently, the linear discriminant analysis effect size (LEfSe) algorithm was performed to identify the bacterial taxa that were most affected by TFDG or EGCG, by using the Huttenhower Lab Galaxy Server, ${ }^{27}$ with an alpha value of 0.05 and a linear discriminant analysis (LDA) score threshold of 4.0. The relative abundances of the significantly affected bacterial taxa were further visualized with a heatmap and clustered with hierarchical clustering, by using the pheatmap package in $\mathrm{R}$. In addition, the significances of all pairwise comparisons among the three treatment groups were conducted by using Student's $t$-test, and the results were visualized in a heatmap. A value of $p<0.05$ was considered statistically significant.

\section{RESULTS AND DISCUSSION}

Comparison of Degradation Kinetics of TFDG and EGCG. The stability of TFDG and EGCG was assessed prior to performing the fermentations. Without the addition of fecal samples, both compounds were incubated in the SIEM medium in the anaerobic chamber at $37{ }^{\circ} \mathrm{C}$ for $48 \mathrm{~h}$. A modest decrease of TFDG and EGCG (20.9\% and $12.4 \%$, respectively) was observed after $48 \mathrm{~h}$, which suggested that these two phenolic compounds were relatively stable in the medium under the experimental conditions. Their degradation by the human gut microbiota was then investigated. TFDG and EGCG were added into the HFS at a final concentration of 50 and $100 \mu \mathrm{mol} / \mathrm{L}$, respectively, and fermented for $48 \mathrm{~h}$ 
Table 1. Tentative Identifications and Dynamic Changes of Metabolites of TFDG and EGCG during Fermentation with HFS

\begin{tabular}{|c|c|c|c|c|c|c|c|c|c|c|c|c|c|}
\hline \multirow{2}{*}{ ID } & \multirow{2}{*}{ Tentative identification ${ }^{a}$} & \multirow{2}{*}{$\begin{array}{l}\text { Chemical } \\
\text { formula }\end{array}$} & \multirow{2}{*}{$\begin{array}{l}{[\mathrm{M}-\mathrm{H}]^{-}} \\
(\mathrm{m} / \mathrm{z})\end{array}$} & \multirow{2}{*}{$\begin{array}{c}\text { RT } \\
(\min )\end{array}$} & \multirow{2}{*}{$\begin{array}{l}\Delta m / z \\
(p p m)\end{array}$} & \multirow{2}{*}{ Source } & \multicolumn{7}{|c|}{ Concentration at different fermentation times $(\mu \mathrm{mol} / \mathrm{L}){ }^{c}$} \\
\hline & & & & & & & $\mathbf{O h}$ & $2 \mathrm{~h}$ & $6 \mathrm{~h}$ & $12 \mathrm{~h}$ & $24 \mathrm{~h}$ & $36 \mathrm{~h}$ & $48 \mathrm{~h}$ \\
\hline \multicolumn{14}{|c|}{ TFs and derivatives } \\
\hline \multirow[t]{2}{*}{ M01 } & Theaflavin-3-gallate & $\mathrm{C}_{36} \mathrm{H}_{28} \mathrm{O}_{16}$ & 715.12970 & 9.64 & -1.06 & TFDG & 0.23 & 0.45 & 0.10 & 0.51 & 0.62 & 0.20 & 0.08 \\
\hline & & & & & & EGCG & n.d. & n.d. & n.d. & n.d. & n.d. & n.d. & n.d. \\
\hline \multirow[t]{2}{*}{ M02 } & Theaflavin-3'-gallate & $\mathrm{C}_{36} \mathrm{H}_{28} \mathrm{O}_{16}$ & 715.13092 & 9.77 & 0.65 & TFDG & 0.14 & 0.22 & 0.11 & 0.34 & 0.30 & 0.15 & n.d. \\
\hline & & & & & & EGCG & n.d. & n.d. & n.d. & n.d. & n.d. & n.d. & n.d. \\
\hline \multirow[t]{2}{*}{ M03 } & Theaflavin $^{\mathrm{b}}$ & $\mathrm{C}_{29} \mathrm{H}_{24} \mathrm{O}_{12}$ & 563.11932 & 9.42 & -0.32 & TFDG & n.d. & n.d. & 0.33 & 1.54 & 2.28 & 1.53 & 0.93 \\
\hline & & & & & & EGCG & n.d. & n.d. & n.d. & n.d. & n.d. & n.d. & n.d. \\
\hline \multirow[t]{2}{*}{ M04 } & Theanaphthoquinone & $\mathrm{C}_{28} \mathrm{H}_{22} \mathrm{O}_{11}$ & 533.10925 & 9.10 & 0.59 & TFDG & n.d. & n.d. & 0.06 & 0.15 & 6.56 & 12.66 & 12.11 \\
\hline & & & & & & EGCG & n.d. & n.d. & n.d. & n.d. & n.d. & n.d. & n.d. \\
\hline \multicolumn{14}{|c|}{ Catechins and diphenylpropanols } \\
\hline \multirow[t]{2}{*}{ M05 } & $\mathrm{EGC}^{\mathrm{b}}$ & $\mathrm{C}_{15} \mathrm{H}_{14} \mathrm{O}_{7}$ & 305.06686 & 5.13 & 0.60 & TFDG & n.d. & n.d. & n.d. & n.d. & n.d. & n.d. & n.d. \\
\hline & & & & & & EGCG & 0.58 & 0.89 & n.d. & n.d. & n.d. & n.d. & n.d. \\
\hline \multirow[t]{2}{*}{ M06 } & 1-( $\left(3^{\prime}, 4^{\prime}, 5^{\prime}-\right.$ Trihydroxyphenyl)-3-(2", $4^{\prime \prime}, 6^{\prime \prime}-$ & $\mathrm{C}_{22} \mathrm{H}_{20} \mathrm{O}_{11}$ & 459.09363 & 6.43 & 0.75 & TFDG & n.d. & n.d. & n.d. & n.d. & n.d. & n.d. & n.d. \\
\hline & trihydroxyphenyl)-propan-2-yl gallate & & & & & EGCG & n.d. & 13.94 & 2.14 & n.d. & n.d. & n.d. & n.d. \\
\hline \multirow[t]{2}{*}{ M07 } & 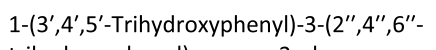 & $\mathrm{C}_{15} \mathrm{H}_{16} \mathrm{O}_{7}$ & 307.08243 & 5.35 & 0.35 & TFDG & n.d. & n.d. & n.d. & n.d. & n.d. & n.d. & n.d. \\
\hline & trihydroxyphenyl)-propan-2-ol & & & & & EGCG & n.d. & 1.49 & n.d. & n.d. & n.d. & n.d. & n.d. \\
\hline \multirow[t]{2}{*}{ M08 } & 1-(3',4'-Dihydroxyphenyl)-3-(2", $4^{\prime \prime}, 6^{\prime \prime}-$ & $\mathrm{C}_{15} \mathrm{H}_{16} \mathrm{O}_{6}$ & 291.08740 & 5.96 & -0.05 & TFDG & n.d. & n.d. & n.d. & n.d. & n.d. & n.d. & n.d. \\
\hline & trihydroxyphenyl)-propan-2-ol & & & & & EGCG & n.d. & 0.86 & 0.26 & 0.08 & n.d. & n.d. & n.d. \\
\hline \multicolumn{14}{|c|}{ Phenylvalerolactones } \\
\hline \multirow[t]{2}{*}{ M09 } & 5- $\left(3^{\prime}, 4^{\prime}, 5^{\prime}\right.$-Trihydroxyphenyl)- $\gamma^{-}$ & $\mathrm{C}_{11} \mathrm{H}_{12} \mathrm{O}_{5}$ & 223.06123 & 5.32 & 0.16 & TFDG & 0.31 & n.d. & n.d. & n.d. & n.d. & n.d. & n.d. \\
\hline & valerolactone & & & & & EGCG & n.d. & 24.91 & 24.1 & 7.74 & 5.55 & 5.82 & 5.00 \\
\hline \multirow[t]{2}{*}{ M10 } & 5-(3',5'-Dihydroxyphenyl)- $\gamma^{-}$ & $\mathrm{C}_{11} \mathrm{H}_{12} \mathrm{O}_{4}$ & 207.06625 & 5.54 & -0.16 & TFDG & n.d. & n.d. & n.d. & n.d. & n.d. & n.d. & n.d. \\
\hline & valerolactone & & & & & EGCG & n.d. & 1.34 & 10.92 & 22.02 & 24.74 & 22.26 & 19.68 \\
\hline
\end{tabular}

${ }^{a}$ Supporting mass spectrometric data can be found in Table S1, Supporting Information. ${ }^{b}$ These identifications were confirmed with authentic standards. ${ }^{c}$ Mean value of compound concentration $(n=4)$; n.d., not detected. The colors range from light blue to dark blue, indicating the range from low to high relative concentration for each metabolite detected in all samples.

under the same conditions. With these concentrations, equal moles of monomeric flavan-3-ol unit as starting materials were applied to facilitate the quantitative comparison of the metabolites. The quantitative changes in TFDG and EGCG concentration throughout the fermentation and the representative extracted ion chromatograms (fermentation times: 0, 2, 6, 24, and $48 \mathrm{~h}$ ) are shown in Figure 2. A faster degradation was observed for EGCG compared to TFDG. After $2 \mathrm{~h}$ of fermentation, compared to the initial concentration $(0 \mathrm{~h}), 72 \%$ of EGCG was degraded by human gut microbiota, while only $44 \%$ of TFDG was degraded. After 12 h, EGCG was almost completely degraded (99.5\%), whereas for TFDG, $68 \%$ was degraded. EGCG was completely depleted after $24 \mathrm{~h}$, whereas $7 \%$ of TFDG remained at the end of fermentation $(48 \mathrm{~h})$. The slower microbial degradation of TFDG can be attributed mainly to its more complex chemical structure. TFDG has a benzotropolone core structure, which may make it less accessible for bacterial enzymes than simpler green tea catechins. Even cleavage of the ester bonds to release gallic acid proceeded more slowly for TFDG than for EGCG.

Phenolic Metabolites Formed during the Fermentation of TFDG and EGCG. The degradation of TFDG and EGCG gave rise to a series of phenolic metabolites in the fermentation samples. A total of 18 metabolites of TFDG and EGCG were tentatively identified. Their corresponding retention times, tentative identifications, molecular formulas, detected mass-to-charge ratios $(\mathrm{m} / \mathrm{z})$ in negative ionization mode, mass errors $(\Delta \mathrm{m} / z)$, and concentrations at different fermentation times are shown in Table 1 . Their $\mathrm{MS}^{2}$ fragment ions, which were used for their tentative identification, are listed in the Table S1, Supporting Information. According to their chemical structures, these metabolites are categorized into four classes, including TFs and derivatives, catechins and diphenylpropanols, phenylvalerolactones, and phenylcarboxylic acids. As the metabolites in the class of "TFs and derivatives" retained their dimeric structure, they were defined as upstream metabolites of TFDG, whereas monomeric phenolic products resulting from the degradation of the benzotropolone moiety were defined as downstream metabolites of TFDG. These two phases of the bacterial metabolism of TFDG are further discussed in the following sections. The concentrations of these metabolites were calculated based on the calibration curves of corresponding authentic standards when available. The metabolites, for which authentic standards were not available, were quantified using the calibration curves of standards with similar structures. Specifically, TFDG was used for the quantification of M01 and M02; TF was used for the quantification of M04; EGCG, EGC, and EC were used for the quantification of M06, M07, and M08, respectively; 5-(4'hydroxyphenyl)valeric acid was used for the quantification of M09-13; 4-hydroxyphenylacetic acid was used for the quantification of M15.

Upstream Metabolism of TFDG. Two theaflavin monogallates (M01 and M02) and TF (M03), which can be formed from TFDG via degalloylation, were detected in samples incubated with TFDG. According to the report of Ganguly et al., TF3G eluted earlier than TF3'G in reversephase liquid chromatography. ${ }^{28}$ Therefore, M01 and M02 were tentatively identified as TF3G and TF3'G, respectively. Chen et al. first reported the degalloylation of TFDG by fecal microbiota after identifying TF, TF3G, and TF3'G as metabolites of TFDG. ${ }^{15,16}$ Our results are in agreement with 


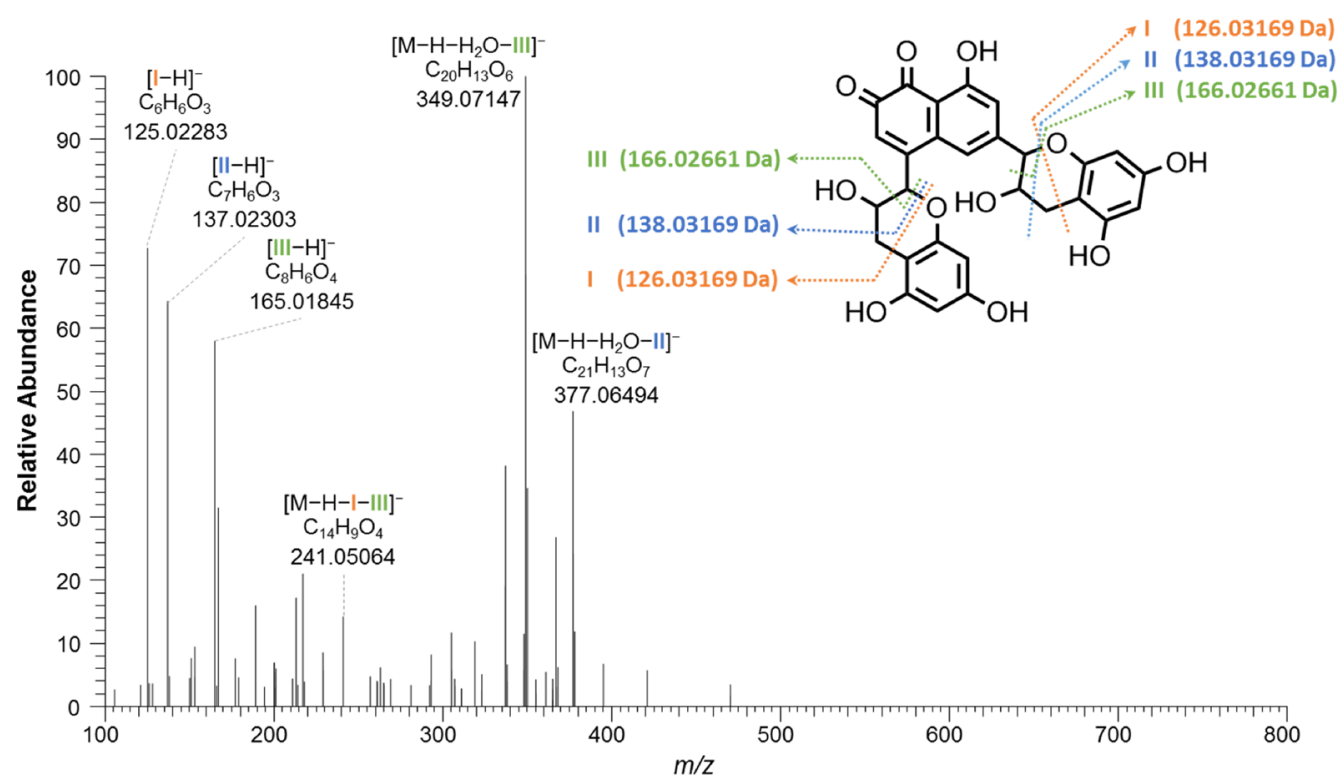

Figure 3. High-resolution HCD fragmentation spectrum $(\mathrm{NCE}=35)$ of theanaphthoquinone (TNQ) in negative ionization mode obtained from UHPLC-Q-Orbitrap-MS. The characteristic peaks are labeled with their corresponding fragmentation pathways as shown in the embedded structure.

these findings, and the cleavage of ester bonds was further confirmed by the identification of gallic acid (M16) and pyrogallol (M18, decarboxylation product of gallic acid). However, the maximum concentrations of theaflavin monogallate and TF (detected at $24 \mathrm{~h}$ ) were found to be relatively low in the TFDG samples during the fermentation, together accounting for approximately $7 \%$ of the initial amount of TFDG.

Instead, a more abundant metabolite (M04) of TFDG was observed in samples incubated with TFDG that was not found in EGCG or blank samples. Its concentration increased during fermentation and peaked at $36 \mathrm{~h}$ with a concentration of 12.7 $\mu \mathrm{mol} / \mathrm{L}$, corresponding to $32 \%$ of the initial amount of TFDG. The extracted ion chromatograms of M04 at various fermentation times are shown in Figure S2, Supporting Information. This compound was detected as its $[\mathrm{M}-\mathrm{H}]^{-}$ ion with a $\mathrm{m} / z$ value of 533.10925 , indicating a molecular formula of $\mathrm{C}_{28} \mathrm{H}_{22} \mathrm{O}_{11}(\Delta \mathrm{m} / z=0.59 \mathrm{ppm})$. The higher energy C-trap dissociation (HCD) fragmentation of this compound gave six major fragment ions with $\mathrm{m} / z$ values of 349.07147 , $125.02283,137.02303,165.01845,377.06494$, and 241.05064 (Figure 3). As neither a deprotonated ion corresponding to gallic acid $(m / z 169)$ nor an ion corresponding to neutral loss of gallic acid $(\mathrm{m} / z$ 381) was observed, M04 is most likely a fully degalloylated TFDG derivative. The MS ${ }^{2}$ fragments at $m$ / $z 125.02283,137.02303$, and 165.01845 correspond to retroDiels-Alder (RDA) fragmentation of the flavan-3-ol heterocyclic ring. ${ }^{29}$ The most abundant fragment ion at $\mathrm{m} / \mathrm{z}$ $349.07147\left(\mathrm{C}_{20} \mathrm{H}_{13} \mathrm{O}_{6}\right)$ corresponds to the combined loss of a $\mathrm{H}_{2} \mathrm{O}$ and $\mathrm{C}_{8} \mathrm{H}_{6} \mathrm{O}_{4}$ (resulting from RDA cleavage) from the parent ion. The fragment ion at $m / z 377.06494\left(\mathrm{C}_{21} \mathrm{H}_{13} \mathrm{O}_{7}\right)$ originates from the loss of a $\mathrm{H}_{2} \mathrm{O}$ and $\mathrm{C}_{7} \mathrm{H}_{6} \mathrm{O}_{3}$ (resulting from RDA cleavage) from the parent ion. The fragment ion at $\mathrm{m} / z$ $241.05064\left(\mathrm{C}_{14} \mathrm{H}_{10} \mathrm{O}_{4}\right)$ may be formed by the neutral loss of a $126 \mathrm{Da}$ fragment and a $166 \mathrm{Da}$ fragment through two successive RDA fissions, which suggests that M04 retains the intact A- and C-rings of TFDG. We hypothesized that its structure was similar to theaflavin $\left(563.11932, \mathrm{C}_{29} \mathrm{H}_{24} \mathrm{O}_{12}\right)$, potentially corresponding to a derivative in which $\mathrm{CH}_{2} \mathrm{O}$ is lost via ring contraction of the seven-membered ring of the benzotropolone moiety.

In order to obtain further mass spectrometric information of this metabolite, TF was fermented with HFS following the same incubation conditions with TFDG, but with a higher final concentration of $500 \mu \mathrm{mol} / \mathrm{L}$ to facilitate acquisition of more in-depth fragmentation data with UHPLC-ESI-IT-MS. As shown in Figure S3, Supporting Information, the metabolite with a $\mathrm{m} / z$ value of 533 (in negative ionization mode) was formed along with the concomitant decrease of TF. We confirmed that this metabolite was identical to metabolite M04 found in the TFDG fermentation due to its identical retention time, accurate mass, and HCD fragmentations as determined by UHPLC-Q-Orbitrap-MS. Thus, the microbial metabolism of both TFDG and TF resulted in formation of M04 as a major metabolite. Furthermore, in UHPLC-ESI-IT-MS, M04 showed collision-induced dissociation (CID) $\mathrm{MS}^{2}$ fragments at $\mathrm{m} / z$ 515, 505, and 471 and CID MS $^{3}$ fragments at $m / z 471,349$, 305, 453, and 165 (Figure S4, Supporting Information), which were in accordance with the spectral data previously reported for theanaphthoquinone (TNQ) by Yassin et al. ${ }^{30}$ TNQ was first reported by Tanaka et al. as an oxidation product of TF formed by polyphenol oxidase or auto-oxidation, and they used NMR spectroscopy to confirm its structure, which includes a 1,2-naphthoquinone moiety. ${ }^{31}$ Thus, an additional model incubation system of TF with tyrosinase was performed to further confirm the identity of this metabolite. It was observed that a major product peak with the same $m / z$ value, fragmentation pattern, and retention time as M04 $(\mathrm{m} / z 533$ and $9.10 \mathrm{~min}$, respectively) was formed after $30 \mathrm{~min}$ of incubation (Figure S5, Supporting Information). This is also in agreement with another study, which reported that TNQ is the main tyrosinase oxidation product of TF. ${ }^{32}$ Therefore, M04 was identified as TNQ. Its chemical structure and highresolution $\mathrm{HCD}$ spectrum and fragmentation pathways are shown in Figure 3. Considering that no TNQ was detected without the addition of fecal samples, we conclude that the 
A

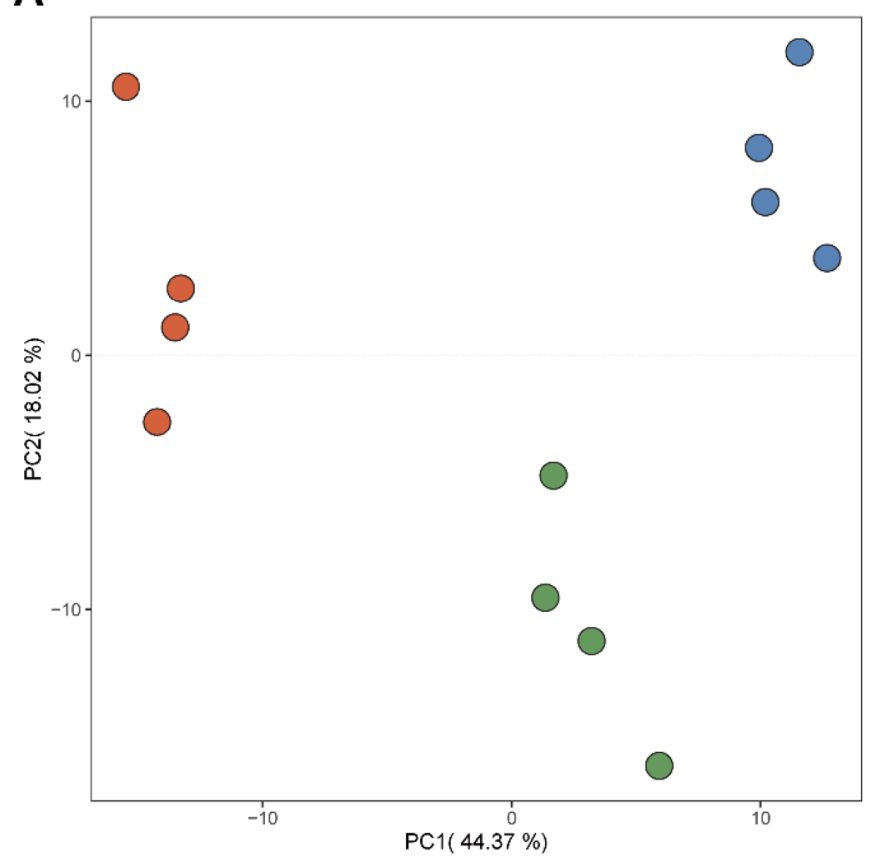

C

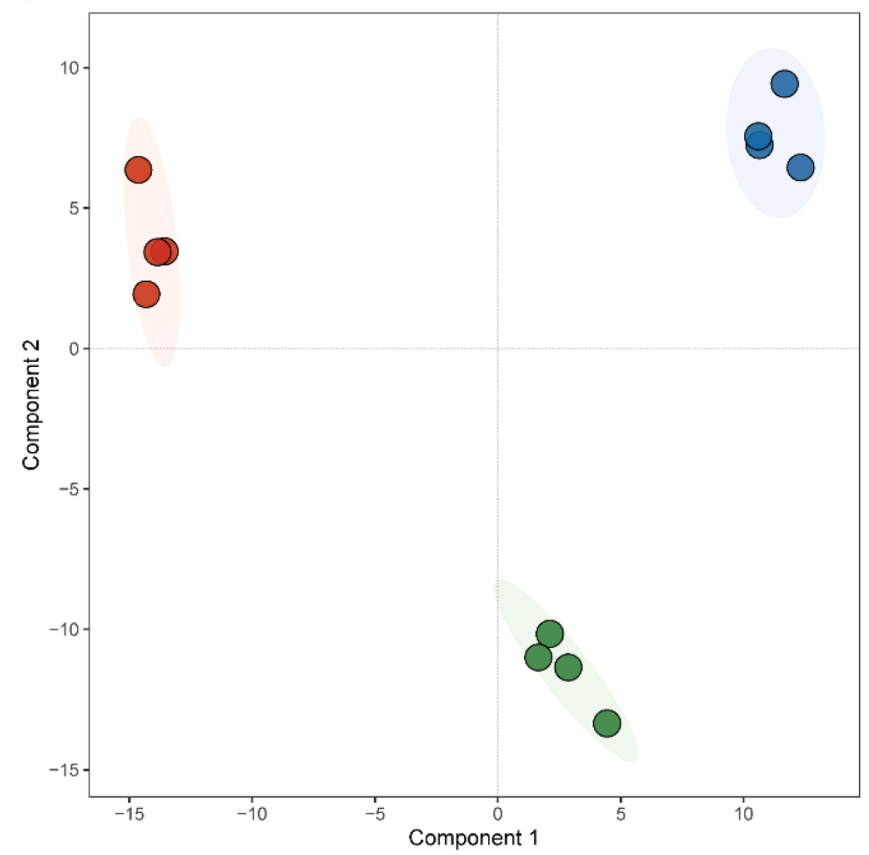

B

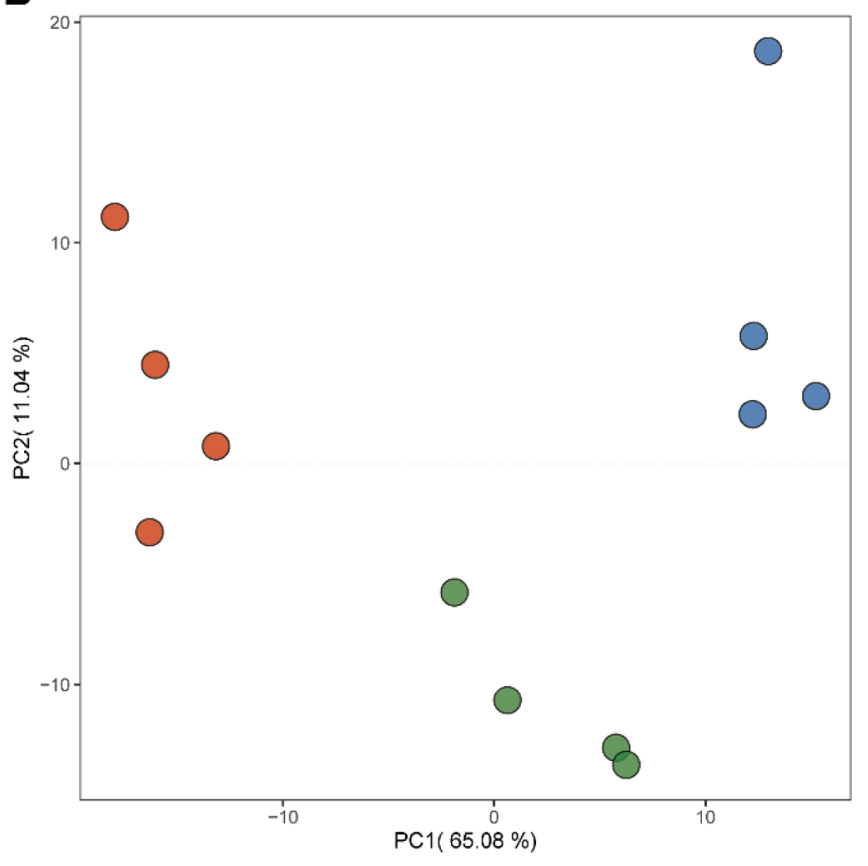

D

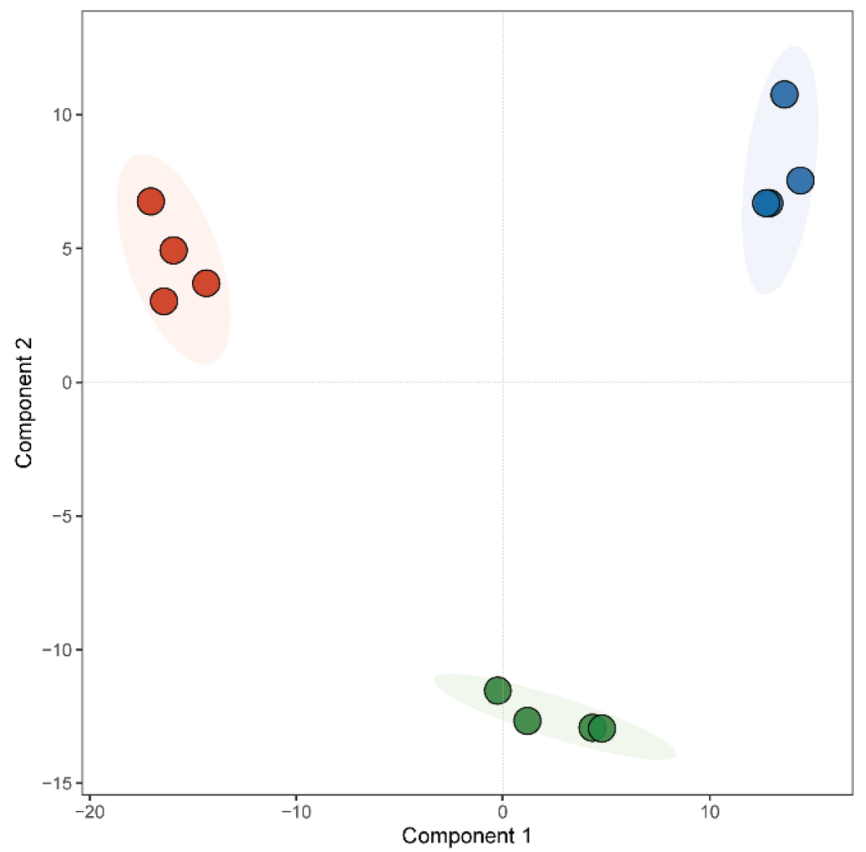

Figure 4. PCoA and PLS-DA score plots of metabolic profiles determined by UHPLC-Q-Orbitrap-MS analysis after $48 \mathrm{~h}$ of fermentation. (A) PCoA score plot based on the data obtained from negative ionization mode; (B) PCoA score plot based on the data obtained from positive ionization mode; (C) PLS-DA score plot based on the data obtained from negative ionization mode; (D) PLS-DA score plot based on the data obtained from positive ionization mode.

conversion of theaflavins to TNQ was mediated by gut microbiota. Anaerobic oxidation widely occurs in the kingdom of bacteria. For example, many bacteria have been reported to possess the ability to oxidize different substrates using azo compounds as the terminal electron acceptor instead of oxygen. ${ }^{33}$ Additionally, anaerobic ammonia oxidation (anammox) plays an important role in the nitrogen cycle and this process can be performed by several species of gut microbiota, such as Candidatus scalindua ${ }^{34}$ and Candidatus brocadia. ${ }^{12}$ Moreover, it was reported that a strictly anaerobic species, Clostridium bryantii sp. nov., can anaerobically oxidize fatty acids to yield acetate and $\mathrm{H}_{2} \cdot{ }^{35}$ We assume that $\mathrm{TF}$, which is formed by degalloylation of TFDG, can be anaerobically oxidized to TNQ by a bacterial oxidase. Further investigation will be necessary to elucidate the microorganisms and enzymes 


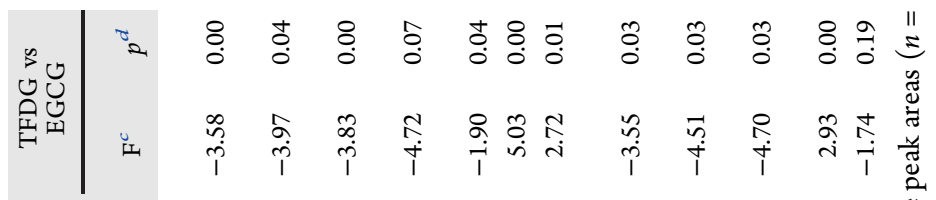

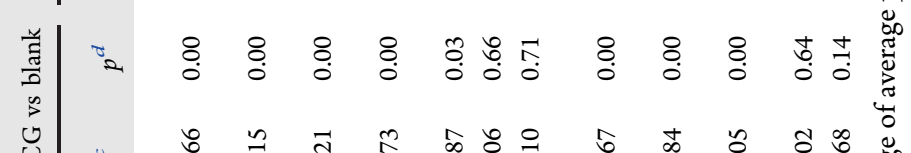

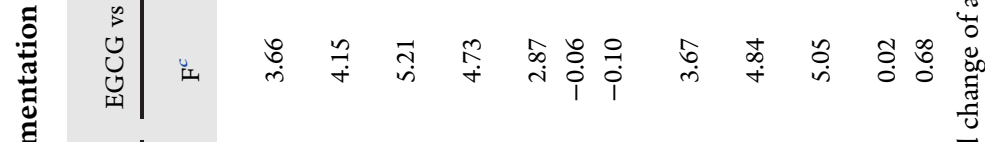

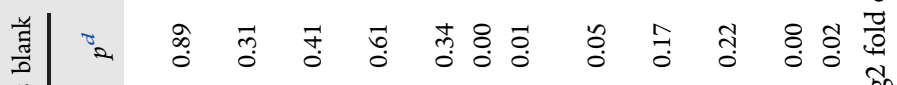

苟 出

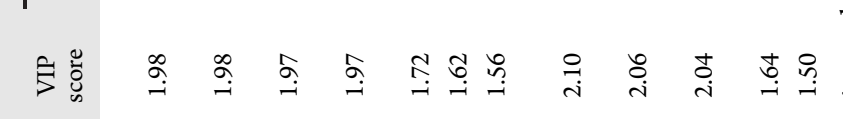

हैं

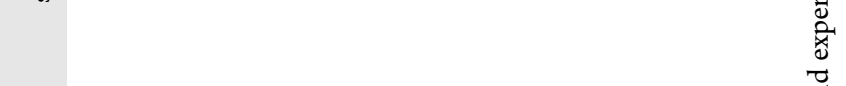

苞

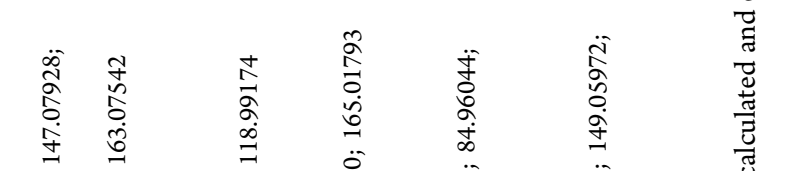

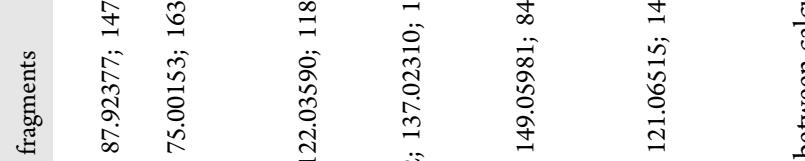

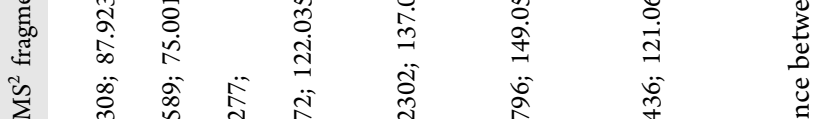

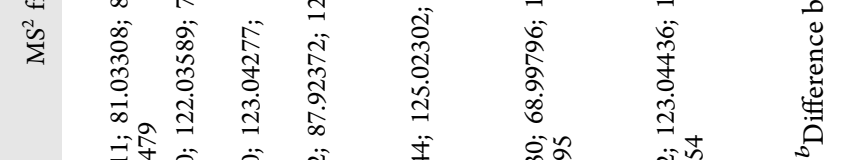

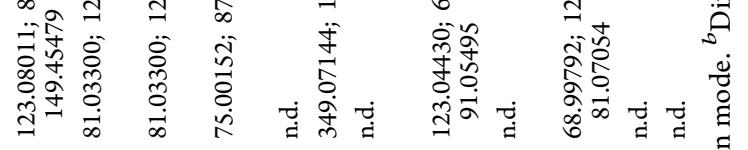

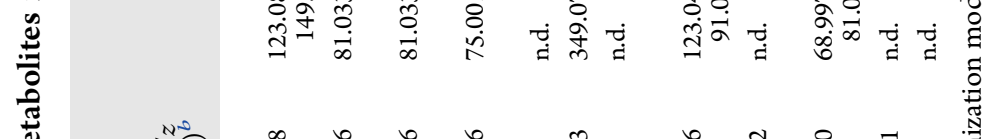

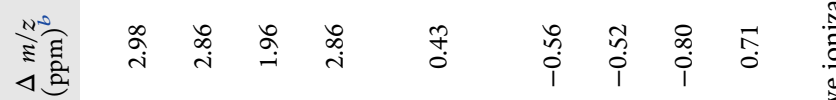

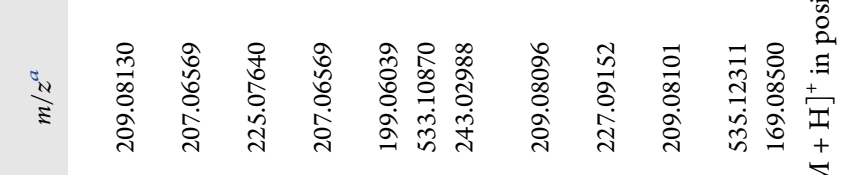

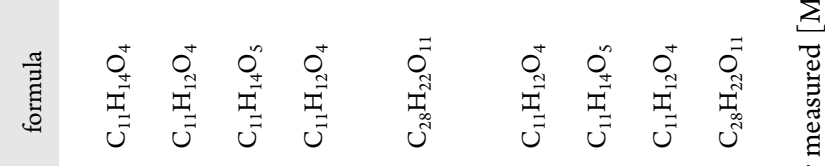

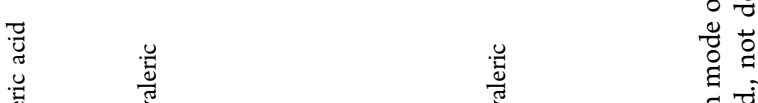

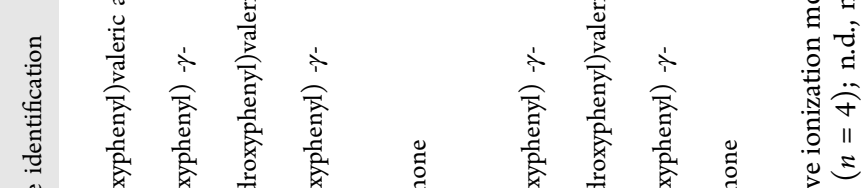

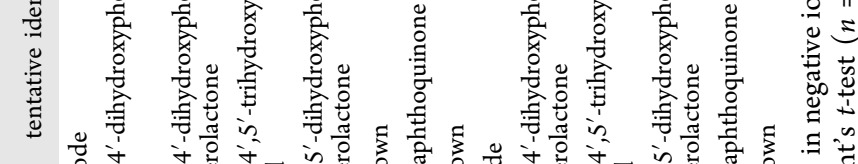

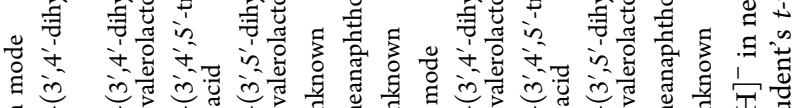

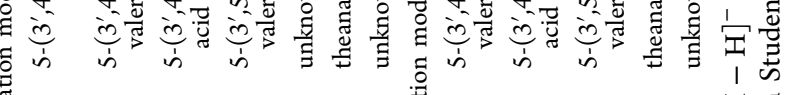

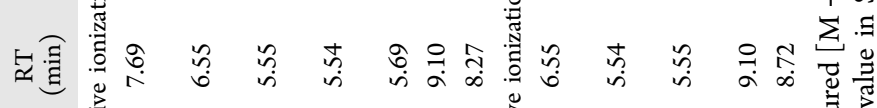

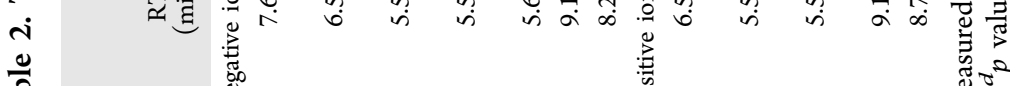

吾 
involved in the anaerobic oxidation process responsible for the formation of this unique metabolite.

Downstream Metabolism of TFDG. Several downstream metabolites of TFDG with a single phenyl moiety were detected, which resulted from degradation of the benzotropolone moiety and subsequent loss of the interflavanic linkage. These metabolites include 5-( $3^{\prime}, 4^{\prime}$-dihydroxyphenyl)- $\gamma$-valerolactone (M11), 5-(3',4'-dihydroxyphenyl)valeric acid (M13), phenylacetic acid (M15), gallic acid (M16), 4-hydroxybenzoic acid (M17), and pyrogallol (M18), as listed in Table 1. Regarding the downstream metabolic fate of TFDG, Gross et al. reported that, when a theaflavin-containing black tea extract was incubated with human fecal slurry, phenylpropionic acid, phenylacetic acid, benzoic acid, and their hydroxylated derivatives would be formed. ${ }^{36}$ However, due to the complex chemical composition of black tea extract, these metabolites could have been derived from other phenolic compounds. Pereira-Caro et al. provided the first direct evidence of the degradation of the theaflavin skeleton by gut microbiota, leading to the formation of theaflavin-related metabolites. ${ }^{8}$ The metabolites reported in that study include 5- $\left(3^{\prime}, 4^{\prime}\right.$-dihydroxyphenyl $)-\gamma$-valerolactone, 5-( $3^{\prime}$-hydroxyphenyl $)-\gamma$-valerolactone, 5-( $3^{\prime}$-hydroxyphenyl)- $\gamma$-hydroxyvaleric acid, 5-(phenyl)$\gamma$-hydroxyvaleric acid, 3-( $3^{\prime}, 4^{\prime}$-dihydroxyphenyl)propionic acid, gallic acid, 3,4-dihydroxybenzoic acid, 3-hydroxybenzoic acid, benzoic acid, and pyrogallol. Qualitatively, our findings are in agreement with these observations, and similar metabolites are well-known colonic degradation products of flavan-3-ol monomers. ${ }^{19}$ Such metabolites were also detected in the fermentation of EGCG in this study, as described in the next section.

Comparison of Downstream Metabolites of TFDG and EGCG. In comparison with the downstream metabolites detected in TFDG samples, a wider variety of phenolic metabolites were found in EGCG samples (Table 1). A small amount of EGC was found at $0 \mathrm{~h}$ in samples incubated with EGCG, which was due to minor EGC impurity $(<0.6 \%)$ in the EGCG standard we used. During the fermentation of EGCG, the concentration of EGC was observed to transitorily increase at $2 \mathrm{~h}$ and completely deplete at $6 \mathrm{~h}$. The increase in EGC was attributed to the degalloylation of EGCG. Three diphenylpropanols (M06-08) corresponding to the C-ring opening products of EGCG, EGC, and EC, respectively, were found in samples incubated with EGCG, but not in TFDG and blank samples. These metabolites were mainly derived from the successive degalloylation, dehydroxylation and C-ring opening of EGCG, as described by Takagaki et al. ${ }^{84}$ Three hydroxyphenylvalerolactones (M09-11), which are formed by A-ring fission of diphenylpropanols, ${ }^{9}$ were identified in samples incubated with EGCG. Further degradation of the hydroxyphenylvalerolactones led to the formation of seven hydroxylated phenylcarboxylic acids (M12-18), which was in line with previous studies. ${ }^{11,14}$ Quantitative analysis revealed that, in general, the fermentation of EGCG led to a higher amount of hydroxylated phenylcarboxylic acids than the fermentation of TFDG (Table 1). Specifically, samples incubated with EGCG contained significantly higher concentrations of hydroxylated phenylvaleric acids (M12 and M13), phenylbutyric acid (M14), gallic acid (M16), and pyrogallol (M18) than those of samples incubated with TFDG. Both samples contained similar concentrations of phenylacetic acid (M15) and 4-hydroxybenzoic acid (M17).
The identification of similar downstream metabolites of TFDG and EGCG indicates that they may share a common microbial degradation pathway, which includes consecutive ester hydrolysis, C-ring opening, A-ring fission, dehydroxylation, and aliphatic chain shortening, as proposed by PereiraCaro et al. ${ }^{8}$ However, these downstream metabolites were generally present at relatively low abundance in samples incubated with TFDG, when compared with the contents observed in EGCG samples. Additionally, we identified TNQ as one of the main metabolites of TFDG. Thus, these results suggest that over a $48 \mathrm{~h}$ of fermentation by human gut microbiota, which is roughly the normal retention time for food components in the colon, hydroxylated phenylcarboxylic acids are not the main metabolites of TFDG. The total concentration of the detected metabolites of TFDG at $48 \mathrm{~h}$ was estimated to be $28.9 \mu \mathrm{mol} / \mathrm{L}$, accounting for $63 \%$ of the initial concentration of TFDG. The remaining TFDG may have been converted to volatile or yet to be identified metabolites, such as the further oxidation products of TNQ.

Comparison of Untargeted Metabolic Profiles of TFDG and EGCG. To further investigate the changes in the metabolic profile upon the fermentation of TFDG and EGCG by gut microbiota, untargeted metabolomics analysis was conducted on the fermentation samples collected at $48 \mathrm{~h}$. Overall, 607 and 793 metabolites were detected in UHPLCHRMS across 12 samples (quadruplicate of TFDG, EGCG, and blank samples at $48 \mathrm{~h}$ ), by using negative and positive ionization modes, respectively. Their peak areas were introduced into $\mathrm{R}$ software for multivariate statistical analysis. First, based on the peak area of metabolites detected in negative and positive ionization modes, two PCoA score plots were made to visualize the differences between the three treatments (Figure 4A,B). Clear segregations were observed for both ionization modes in the PCoA plots, which explained a total of $62.4 \%$ and $76.1 \%$ of variances, respectively. When compared with the blank samples, the PCoA score plot showed a significant shift of the general metabolic profile after TFDG or EGCG treatment. There is also clear distinction between TFDG and EGCG treatments. In order to identify the metabolites distinguishing the three groups, PLS-DA was performed for both ionization modes, as shown in Figure 4C,D. In the cross-validation of the PLS-DA model derived from negative ionization, the model fit $\left(R^{2}\right)$ and predictiveness $\left(Q^{2}\right)$ values were found to be 0.98 and 0.94 , respectively, suggesting good fitness and predictive power of this model. For positive ionization, $R^{2}$ and $Q^{2}$ values were 0.98 and 0.87 , respectively. In these supervised models, complete separation among the three treatment groups was observed in both ionization modes. Next, VIP scores were calculated to evaluate the contribution of each metabolite to the total variance in the PLS-DA models. Based on the results of PLS-DA modeling, we screened for the metabolites distinguishing the three groups. The screening criteria included (i) high contribution to sample classification in PLS-DA (VIP score > 1.5); (ii) considerable peak area change in the pairwise comparison among three groups (fold change > 3); and (iii) statistically significant change in the pairwise comparison among three groups $(p<$ 0.05 in Student's $t$-test). With these criteria, a total of 12 metabolites were selected, 7 in negative ionization mode and 5 in positive ionization mode. These metabolites are summarized in Table 2 with their corresponding retention time, tentative identification, molecular formulas, $\mathrm{m} / z$ values, mass errors, 

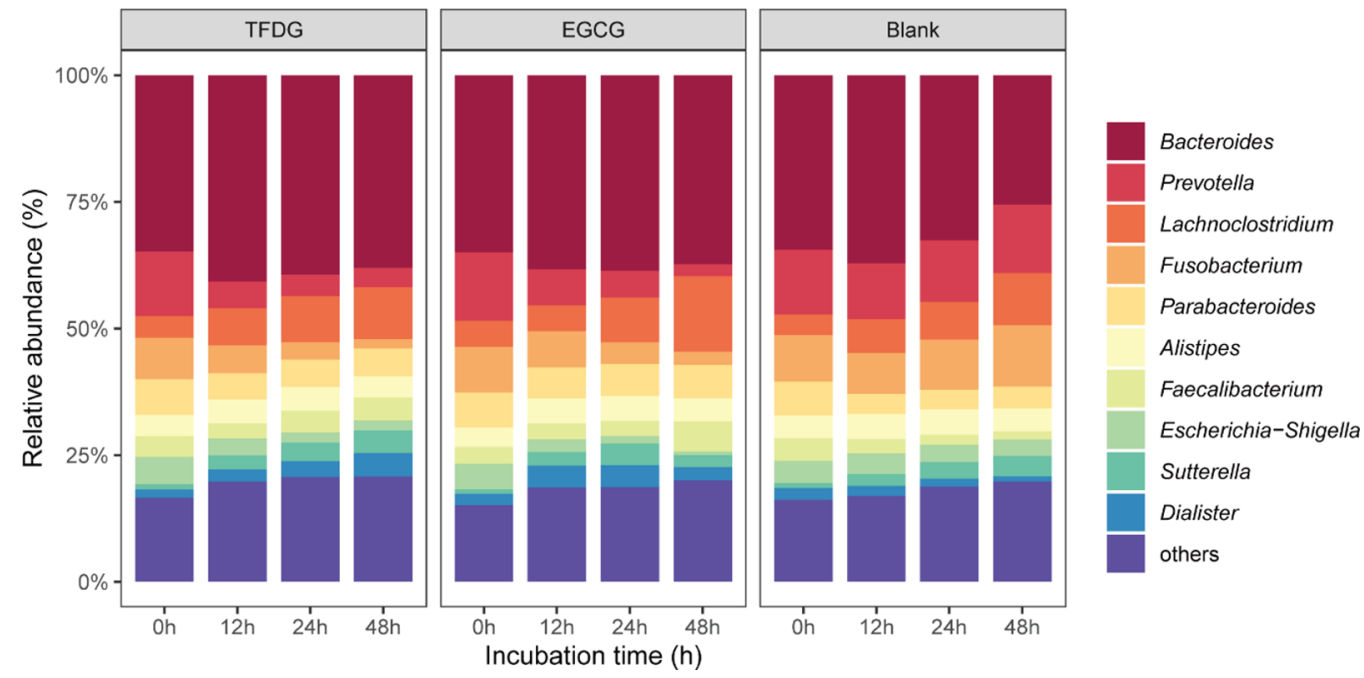

Figure 5. Relative abundances of the most abundant bacterial taxa at genus level during fermentation with TFDG, EGCG, and blank.

fragment ions, and pairwise comparison fold changes and $p$ values.

Among these distinguishing metabolites, 5- $\left(3^{\prime}, 4^{\prime}\right.$ dihydroxyphenyl)valeric acid, 5-( $3^{\prime}, 4^{\prime}$-dihydroxyphenyl $)-\gamma$-valerolactone, 5- $\left(3^{\prime}, 4^{\prime}, 5^{\prime}\right.$-trihydroxyphenyl)valeric acid, 5- $\left(3^{\prime}, 5^{\prime}\right.$ dihydroxyphenyl)- $\gamma$-valerolactone, and TNQ were derived from the degradation of TFDG or EGCG. The three remaining distinguishing metabolites could not be identified but may be derived from other compounds present in the fermentation samples, e.g., microbial metabolites of medium constituents. The effects of TFDG or EGCG treatment on seemingly unrelated bacterial metabolic pathways need to be further explored by identification of these unknown metabolites. Nevertheless, the data generated from the untargeted UHPLCQ-Orbitrap-MS metabolome profiling approach implied that TFDG and EGCG considerably altered the metabolic profiles of fecal microbiota, as shown in Figure 4. This alteration may be attributed to modulation of the microbial community as well as changes in metabolic activity of fecal microbiota. Our findings hereby emphasize the potential impact of TFDG and EGCG on the colonic environment and therefore the health status of the human host.

Comparison of Gut Microbiota Composition. Microbiota community compositions in the fermentation samples at $0,12,24$, and $48 \mathrm{~h}$ were assessed by Illumina high-throughput sequencing of bacterial $16 \mathrm{~S}$ rRNA genes. After merging and filtration, a total of 6,123,109 reads were obtained for the microbiome analysis across all samples. These sequencing reads were clustered into 302 OTUs with $97 \%$ similarity. Of these OTUs, nine phyla were observed, including Bacteroidetes, Firmicutes, Proteobacteria, Fusobacteria, Actinobacteria, Cyanobacteria, Lentisphaerae, Verrucomicrobia, and Tenericutes (Figure S6, Supporting Information). At the genus level, a total of 289 genera were identified, with Bacteroides, Prevotella, Lachnoclostridium, Fusobacterium, Parabacteroides, Alistipes, Faecalibacterium, Escherichia-Shigella, Sutterella, and Dialister as the top 10 most abundant ones. Their relative abundances and shifts over the fermentation time across the three groups are depicted in Figure 5. In general, the initial bacterial community compositions were similar in all three treatment groups. During fermentation, the bacterial community compositions in the samples incubated with TFDG and EGCG were comparable, both showing an increase in Bacteroides and Lachnoclostridium and a decrease in Prevotella. On the contrary, a decrease in Bacteroides and an increase in Fusobacterium were observed in blank samples.

Further comparison of the bacterial community composition among all samples was performed by using hierarchical clustering analysis and PCA, as shown in Figure 6. In hierarchical clustering analysis, the fecal microbiota compositions were clustered into four groups: (i) TFDG, EGCG and blank samples at $0 \mathrm{~h}$; (ii) TFDG and EGCG samples at 12 and $24 \mathrm{~h}$; (iii) TFDG and EGCG samples at $48 \mathrm{~h}$; (iv) blank samples at 12, 24, and $48 \mathrm{~h}$ (Figure 6A). Similarly, as shown in Figure 6B, PCA shows that the bacterial community composition changed in response to treatment of TFDG and EGCG. Particularly, after $48 \mathrm{~h}$, there was a distinct clustering of microbiota composition between the samples incubated with tea phenolic (TFDG or EGCG) and blank samples. Moreover, both hierarchical cluster analyses and PCA scatter plot suggested that TFDG and EGCG had a similar modulatory effect on the gut microbiota composition after $48 \mathrm{~h}$. It should be noted that, during the in vitro fermentation, changes in microbiota composition were observed over time, even without the addition of tea phenolics. This is most likely caused by the fact that the sophisticated colonic environment could not be precisely mimicked in vitro. Nevertheless, the gut microbiota modulatory effects of TFDG and EGCG are apparent when compared to blank samples.

To gain insight in which bacteria were most affected by TFDG and EGCG after $48 \mathrm{~h}$ of fermentation, LEfSe analysis with an LDA score threshold of $>4.0$ was performed. A total of 34 bacterial taxa were identified to be differentially enriched in the three treatment groups. Their phylogenetic relationships and LDA scores are shown in a cladogram (Figure 7A) and a histogram (Figure 7B), respectively. Comparison with the blank revealed that 8 taxa were increased by TFDG, 12 taxa were increased by EGCG, and 14 taxa were decreased by both TFDG and EGCG, as reflected in a heatmap based on their scaled relative abundance (Figure $7 \mathrm{C}$ ). Significance of the pairwise comparisons of the specific bacterial taxon among the three groups is depicted in an adjacent heatmap (Figure 7C). It is notable to mention that, at the genus level, both TFDG and EGCG significantly promoted Bacteroides, Faecalibacterium, Parabacteroides, and Bifidobacterium $(p<0.05)$ and significantly inhibited Prevotella and Fusobacterium $(p<0.05)$. In this 
A

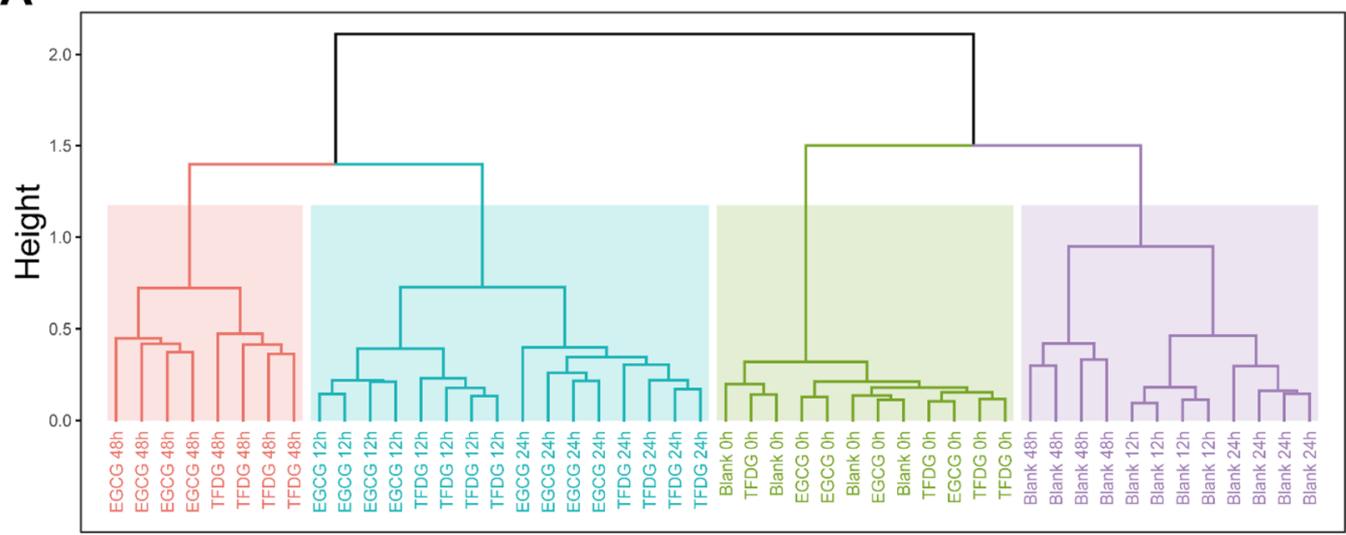

B

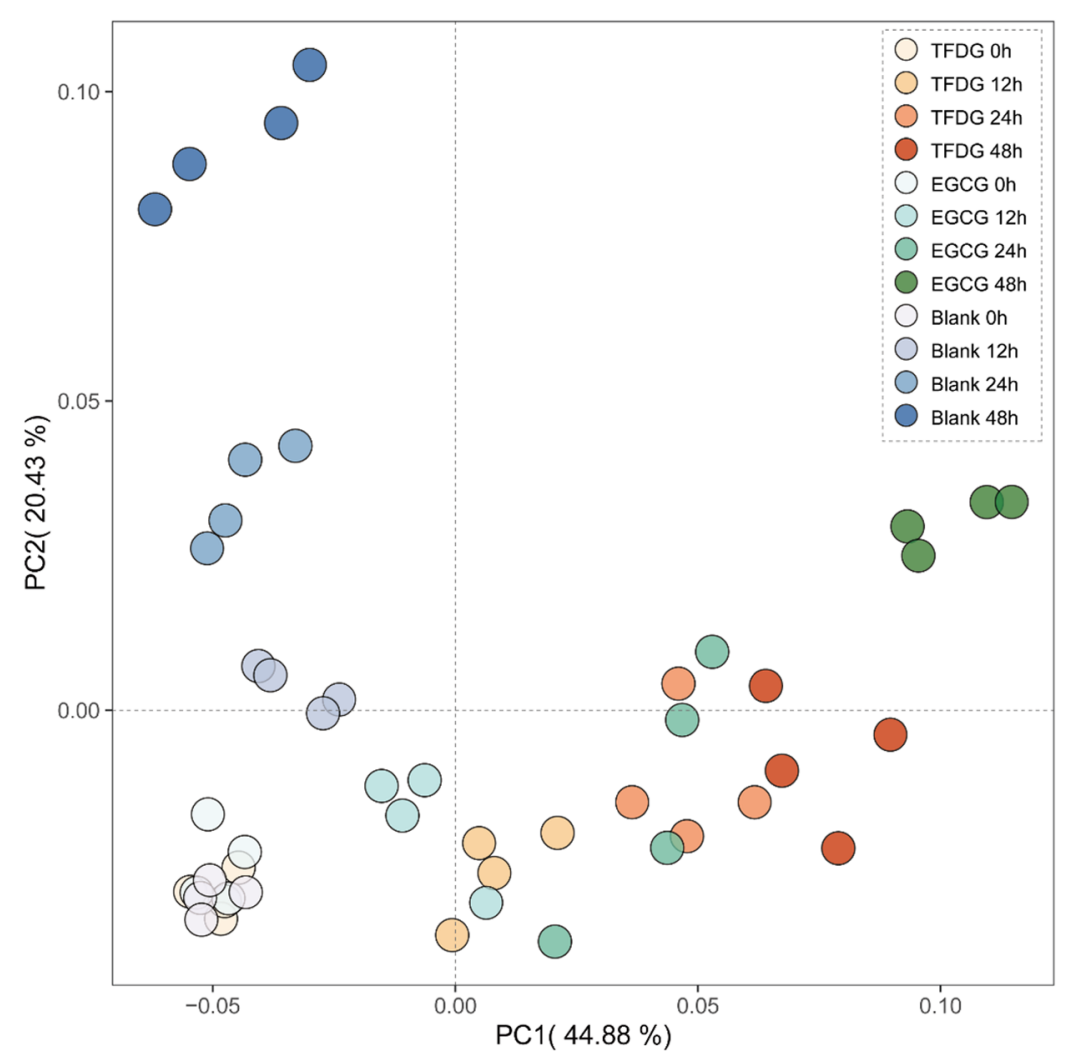

Figure 6. Hierarchical clustering analysis (A) and PCA score plots (B) based on relative abundance of bacterial 16S gene OTUs of microbial communities in the samples from different fermentation treatments and times.

study, we identified eight Bacteroides species with average relative abundance above $1 \%$. Most of these species were significantly increased by TFDG and/or EGCG, with the exceptions of Bacteroides ovatus and Bacteroides cellulosilyticus (Figure S7, Supporting Information). The genus Bacteroides is one of the dominant genera in human gut microbiota, which constitutes $20-40 \%$ of the human colonic bacteria and imparts substantial metabolic, immunologic, and defensive functions in the gastrointestinal tract. ${ }^{37}$ An in vivo study demonstrated that long-term treatment with green tea phenolics would lead to colonic enrichment of Bacteroides in rats. ${ }^{38}$ Increased abundance of Bacteroides species in the colon can contribute to improved metabolism of undigested nutrients and attenuation of colon inflammation. ${ }^{39}$ Therefore, our results on the promotion of Bacteroides species by TFDG and EGCG indicate that tea consumption may support a healthier colonic environment.

In addition, several bacterial taxa were revealed to be differentially affected by TFDG and EGCG. For example, Dialister was only significantly promoted by TFDG $(p<0.01)$, Clostridium symbiosum was only significantly promoted by EGCG $(p<0.05)$, and Escherichia coli was only significantly inhibited by EGCG $(p<0.05)$. It is well known that catechins exhibit antimicrobial activities against the growth of some pathogens including E. coli and Clostridium perfringens. ${ }^{40}$ Lee et al. found that E. coli was more susceptible to bacterial metabolites of catechins than to their parent compounds. ${ }^{41}$ Therefore, a more potent inhibitory effect of EGCG against $E$. coli compared to TFDG observed in this study could be explained by the formation of a higher amount of hydroxylated phenylcarboxylic acids after EGCG fermentation as described 
A
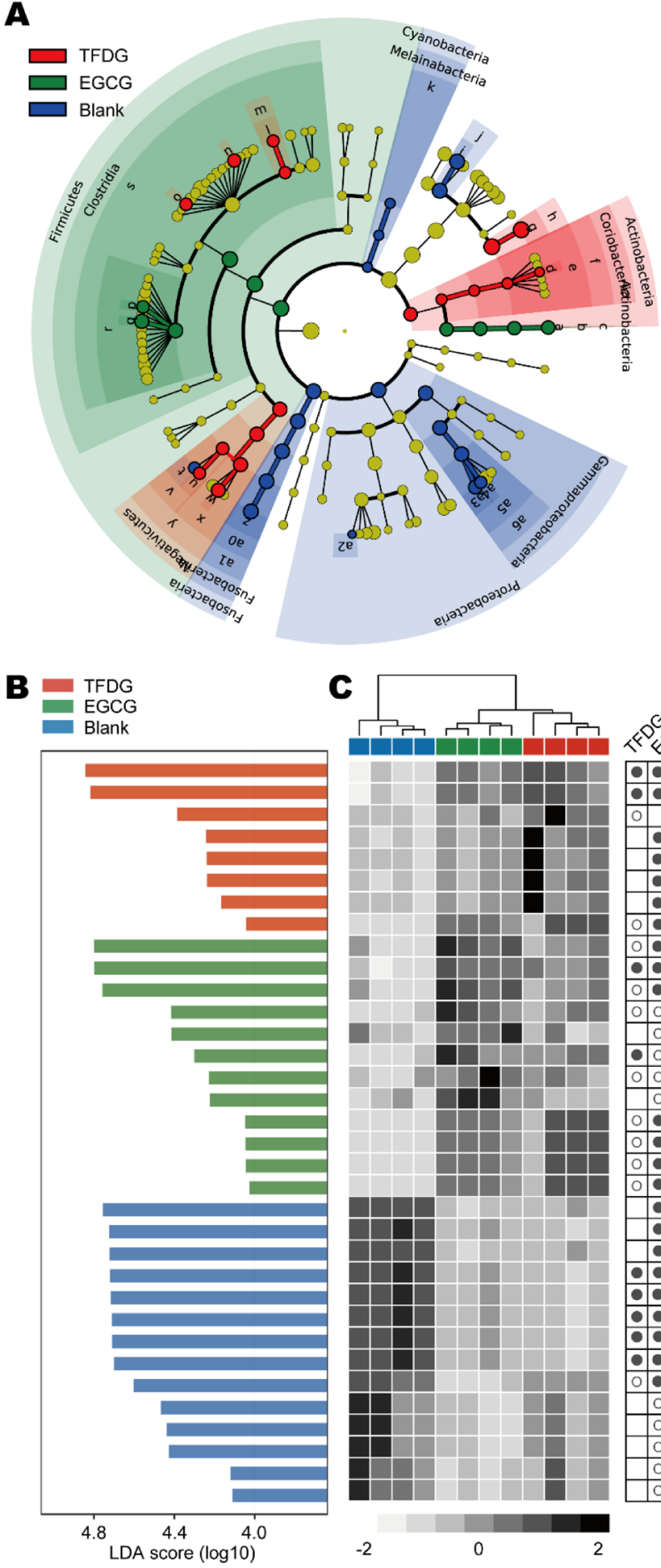

a: Bifidobacterium

b: Bifidobacteriaceae

d: Olsenella

e: Coriobacteriaceae

g: Bacteroides

h: Bacteroidacea

i: Prevotella

j: Prevotellaceae

I: Eubacterium

m: Eubacteriace

n: Dorea

o: Roseburia

q. Faecalibacterium

r: Ruminococcacea

s: Clostridiales

i. Slostridiales

u: Phascolarctobacterium

v: Acidaminococcacea

w: Dialister

x: Veillonellaceae

y: Selenomonadales

z: Fusobacterium

a0: Fusobacteriaceae

a1: Fusobacteriales

a2: Achromobacter

a4: Klebsiella

as: Enterobacteriaceae

a6: Enterobacteriales

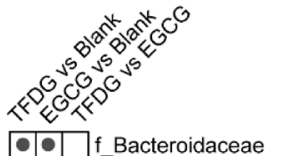

- Df_Bacteroidacea

\begin{tabular}{llll}
\hline & & g_Bacteroides \\
\hline 0 & & s_Bacteroides uniformis
\end{tabular}

- O_Selenomonadales

- g_Dialister

- C_Negativicutes

- f_Veillonellaceae

O - P_Actinobacteria

- C_Clostridia

- 0 p_Firmicutes

O- -0 O_Clostridiales

O 0 f_Ruminococcaceae

\begin{tabular}{lll} 
& 0 & s_Clostridium symbiosum \\
\hline
\end{tabular}

- 1 g_Faecalibacterium

\begin{tabular}{lllll} 
& 0 & g_Faecalibacterium \\
\hline 0 & 0 & & s_Parabacteroides distasonis
\end{tabular}

\begin{tabular}{rlll} 
& 0 & 0 & S_Bacteroides plebeius \\
\hline
\end{tabular}

o - g Bifidobacterium

- C_Actinobacteria

- f_Bifidobacteriaceae

\begin{tabular}{lll}
0 & f_Bifidobacteriaceae \\
\hline & o Bifidobacteriales
\end{tabular}

\begin{tabular}{lll} 
- & o_Bifidobacteriales \\
\hline & f_Prevotellaceae
\end{tabular}

- f_Fusobacteriaceae

- g_Prevotella

- S Fusobacterium varium

- D_Fusobacteria

- g_Fusobacterium

- C_Fusobacteriia

- DofFusobacteriales

- P Proteobacteria

(

O O C_Gammaproteobacteria

o 0 f_Enterobacteriacea

o S_Escherichia coli

O g_Escherichia-Shigella

Figure 7. Comparison of taxonomic abundance between TFDG, EGCG, and blank samples by linear discriminant analysis effect size (LEfSe) analysis. (A) Cladogram representing the phylogenetic relationships among the significantly different taxa with an LDA score > 4.0. From inside out, black rings represent the taxonomic ranks of phylum, class, order, family, and genus. Nodes and branches in red, green, and blue represent significant enrichment in a bacterial taxon by the corresponding treatment; those in yellow were not found to be significantly enriched by any specific treatment. (B) LDA scores of each discriminant bacterial taxon. (C) Left heatmap, scaled relative abundance of discriminant bacterial taxa; right heatmap, significant pairwise comparisons of the three groups. Open circles indicate $p$ value $<0.05$, whereas closed circles indicate $p$ value $<$ 0.01 .

in the Phenolic Metabolites Formed during the Fermentation of TFDG and EGCG section.

The fecal microbiota performed complex conversions of the compounds present in the medium, including TFDG and EGCG. The alteration in the bacterial composition induced by
TFDG and EGCG subsequently resulted in further divergence of the general metabolic profiles, which was demonstrated in Table 2 and Figure 4. More in-depth studies are required to explore the microorganisms mediating the bioconversion of theaflavins to $\mathrm{TNQ}$ and the microorganisms and metabolic 
machinery responsible for the downstream metabolism of both theaflavins and catechins. In addition, it should be noted that the fecal inoculum used in this study originated from pooled fecal material from four volunteers. Recent studies have indicated that inter-individual variation in microbiota composition can prompt differences in metabolism of dietary bioactive small molecules, like phenolic compounds in coffee ${ }^{42}$ and anthocyanins in fruits. ${ }^{43}$ Thus, it would be also interesting to further investigate the extent of inter-individual variation in the gut microbial metabolism of tea phenolics, such as the bioconversion of theaflavins to TNQ.

In conclusion, our results show that human gut microbiota convert TFDG into a number of metabolites, including TNQ as one of its main metabolites, over $48 \mathrm{~h}$ of fermentation. When compared with the metabolism of EGCG, TFDG metabolism yielded a distinctive overall metabolite profile, slower degradation rate, and lower concentrations of downstream metabolites. Despite these differences in their metabolism, EGCG and TFDG demonstrated similar effects on gut microbiota composition, including the promotion of Bacteroides, Faecalibacterium, Parabacteroides, and Bifidobacterium, and the inhibition of Prevotella and Fusobacterium. These findings indicate that, even though the metabolic fates of TFDG and EGCG are distinctly different, their gut microbiota composition modulatory effects are similar. The integrated metabolite and microbiome profiling approach used in this study resulted in new insights on the reciprocal interactions between TFDG and gut microbiota. As TFDG with its benzotropolone moiety is representative for one of the main classes of black tea phenolics, our results may be extrapolated to a large percentage of the black tea phenolic composition. Thereby, the presented comparison of TFDG and EGCG contributes to a more comprehensive understanding of the health-promoting effects of black and green tea.

\section{ASSOCIATED CONTENT}

\section{SI Supporting Information}

The Supporting Information is available free of charge at https://pubs.acs.org/doi/10.1021/acs.jafc.0c06622.

Untargeted metabolomics workflow used in Compound Discoverer (Figure S1); mass spectrometric data of TFDG and EGCG metabolites tentatively identified by UHPLC-Q-Orbitrap-MS (Table S1); extracted ion chromatograms of M04 in negative ion mode UHPLCQ-Orbitrap-MS of TFDG fermentation samples at different fermentation times (Figure S2); extracted ion chromatograms of theaflavin and theanaphthoquinone in negative ion mode UHPLC-ESI-IT-MS of theaflavin fermentation samples at different fermentation times (Figure S3); fragmentation spectra of theanaphthoquinone in negative ion mode UHPLC-ESI-IT-MS (Figure S4); UHPLC-ESI-IT-MS negative ion mode $(\mathrm{m} / z$ 1001000) base peak chromatogram of TF incubated with tyrosinase for $30 \mathrm{~min}$ (Figure S5); relative abundances of the most abundant bacterial taxa at phylum level during fermentation with TFDG, EGCG, and blank (Figure S6); and comparison of the relative abundance of Bacteroides species with average relative abundance above $1 \%$ across all fermentation samples (Figure S7) (PDF)

\section{AUTHOR INFORMATION}

\section{Corresponding Author}

Jean-Paul Vincken - Laboratory of Food Chemistry, Wageningen University, 6700 AA Wageningen, The Netherlands; ○ orcid.org/0000-0001-8540-4327; Phone: +31-317482234; Email: jean-paul.vincken@wur.nl

\section{Authors}

Zhibin Liu - Laboratory of Food Chemistry, Wageningen University, 6700 AA Wageningen, The Netherlands; Institute of Food Science \& Technology, Fuzhou University, Fuzhou 350108, P.R. China

Wouter J. C. de Bruijn - Laboratory of Food Chemistry, Wageningen University, 6700 AA Wageningen, The Netherlands; 이이.orcido000-0003-0564-9848

Marieke E. Bruins - Food \& Biobased Research, Wageningen University \& Research, 6700 AA Wageningen, The Netherlands

Complete contact information is available at:

https://pubs.acs.org/10.1021/acs.jafc.0c06622

\section{Funding}

Z.L. acknowledges the financial support from the China Scholarship Council (CSC).

\section{Notes}

The authors declare no competing financial interest.

\section{ACKNOWLEDGMENTS}

The authors thank Mark Sanders of the Laboratory of Food Chemistry, Wageningen University, for his excellent technical assistance.

\section{ABBREVIATIONS USED}

$\mathrm{ACN}$, acetonitrile; CID, collision-induced dissociation; EC, epicatechin; ECG, epicatechin-3-gallate; EGC, epigallocatechin; EGCG, epigallocatechin-3-gallate; ESI, electrospray ionization; FWHM, full width at half maximum; HCD, higher energy C-trap dissociation; HFS, human fecal suspension; LDA, linear discriminant analysis; LEfSe, linear discriminant analysis effect size; OTU, operational taxonomic unit; PCA, principal component analysis; $\mathrm{PCoA}$, principal coordinates analysis; PLS-DA, partial least-squares discriminant analysis; RDA, retro-Diels-Alder; SIEM, standard ileal efflux medium; $\mathrm{TF}$, theaflavin; TF3G, theaflavin-3-gallate; TF3'G, theaflavin$3^{\prime}$-gallate; TFDG, theaflavin-3,3'-digallate; TNQ theanaphthoquinone; UHPLC-Q-Orbitrap-MS, ultra-high performance liquid chromatography coupled to hybrid quadrupole Orbitrap mass spectrometry; IT-MS, ion trap mass spectrometry; VIP, variable importance in projection

\section{REFERENCES}

(1) Wang, D.; Kurasawa, E.; Yamaguchi, Y.; Kubota, K.; Kobayashi, A. Analysis of glycosidically bound aroma precursors in tea leaves. 2. Changes in glycoside contents and glycosidase activities in tea leaves during the black tea manufacturing process. J. Agric. Food Chem. 2001, 49, 1900-1903.

(2) Drynan, J. W.; Clifford, M. N.; Obuchowicz, J.; Kuhnert, N. The chemistry of low molecular weight black tea polyphenols. Nat. Prod. Rep. 2010, 27, 417-462.

(3) Verloop, A. J. W.; Vincken, J.-P.; Gruppen, H. Peroxidase can perform the hydroxylation step in the "oxidative cascade" during oxidation of tea catechins. J. Agric. Food Chem. 2016, 64, 8002-8009. 
(4) Verloop, A. J. W.; Gruppen, H.; Bisschop, R.; Vincken, J.-P. Altering the phenolics profile of a green tea leaves extract using exogenous oxidases. Food Chem. 2016, 196, 1197-1206.

(5) Mulder, T. P. J.; van Platerink, C. J.; Wijnand Schuyl, P. J.; van Amelsvoort, J. M. M. Analysis of theaflavins in biological fluids using liquid chromatography-electrospray mass spectrometry. J. Chromatogr. B: Biomed. Sci. Appl. 2001, 760, 271-279.

(6) Silva, B. M.; Oliveira, P. F.; Casal, S.; Alves, M. G.; Dias, T. R. Promising potential of dietary (poly) phenolic compounds in the prevention and treatment of diabetes mellitus. Curr. Med. Chem. 2017, 24, 334-354.

(7) Stalmach, A.; Mullen, W.; Steiling, H.; Williamson, G.; Lean, M. E. J.; Crozier, A. Absorption, metabolism, and excretion of green tea flavan-3-ols in humans with an ileostomy. Mol. Nutr. Food Res. 2010, 54, 323-334.

(8) Pereira-Caro, G.; Moreno-Rojas, J. M.; Brindani, N.; del Rio, D.; Lean, M. E. J.; Hara, Y.; Crozier, A. Bioavailability of black tea theaflavins: absorption, metabolism, and colonic catabolism. J. Agric. Food Chem. 2017, 65, 5365-5374.

(9) Mena, P.; Bresciani, L.; Brindani, N.; Ludwig, I. A.; Pereira-Caro, G.; Angelino, D.; Llorach, R.; Calani, L.; Brighenti, F.; Clifford, M. N.; Gill, C. I. R.; Crozier, A.; Curti, C.; del Rio, D. Phenyl- $\gamma$ valerolactones and phenylvaleric acids, the main colonic metabolites of flavan-3-ols: Synthesis, analysis, bioavailability, and bioactivity. Nat. Prod. Rep. 2019, 36, 714-752.

(10) Cueva, C.; Gil-Sánchez, I.; Ayuda-Durán, B.; GonzálezManzano, S.; González-Paramás, A. M.; Santos-Buelga, C.; Bartolomé, B.; Moreno-Arribas, M. An integrated view of the effects of wine polyphenols and their relevant metabolites on gut and host health. Molecules 2017, 22, 99.

(11) Chen, H.; Sang, S. Biotransformation of tea polyphenols by gut microbiota. J. Funct. Foods 2014, 7, 26-42.

(12) Liu, Z.; de Bruijn, W. J. C.; Bruins, M. E.; Vincken, J.-P. Reciprocal interactions between epigallocatechin-3-gallate (EGCG) and human gut microbiota in vitro. J. Agric. Food Chem. 2020, 68, 9804-9815.

(13) Dueñas, M.; Cueva, C.; Muñoz-González, I.; Jiménez-Girón, A.; Sánchez-Patán, F.; Santos-Buelga, C.; Moreno-Arribas, M.; Bartolomé, B. Studies on modulation of gut microbiota by wine polyphenols: from isolated cultures to omic approaches. Antioxidants 2015, 4, 1-21.

(14) Takagaki, A.; Nanjo, F. Biotransformation of (-)-epigallocatechin and (-)-gallocatechin by intestinal bacteria involved in isoflavone metabolism. Biol. Pharm. Bull. 2015, 38, 325-330.

(15) Chen, H.; Parks, T. A.; Chen, X.; Gillitt, N. D.; Jobin, C.; Sang, S. Structural identification of mouse fecal metabolites of theaflavin 3 , $3^{\prime}$-digallate using liquid chromatography tandem mass spectrometry. J. Chromatogr. A 2011, 1218, 7297-7306.

(16) Chen, H.; Hayek, S.; Rivera Guzman, J.; Gillitt, N. D.; Ibrahim, S. A.; Jobin, C.; Sang, S. The microbiota is essential for the generation of black tea theaflavins-derived metabolites. PLoS One 2012, 7, No. e51001.

(17) Chen, T.; Liu, A. B.; Sun, S.; Ajami, N. J.; Ross, M. C.; Wang, H.; Zhang, L.; Reuhl, K.; Kobayashi, K.; Onishi, J. C.; Zhao, L.; Yang, C. S. Green Tea Polyphenols Modify the Gut Microbiome in $d b / d b$ Mice as Co-Abundance Groups Correlating with the Blood Glucose Lowering Effect. Mol. Nutr. Food Res. 2019, 63, 1801064.

(18) Gosslau, A.; Li, S.; Zachariah, E.; Ho, C.-T. Therapeutic Connection Between Black Tea Theaflavins and Their Benzotropolone Core Structure. Curr. Pharmacol. Rep. 2018, 4, 447-452.

(19) Liu, Z.; Bruins, M. E.; Ni, L.; Vincken, J.-P. Green and black tea phenolics: Bioavailability, transformation by colonic microbiota, and modulation of colonic microbiota. J. Agric. Food Chem. 2018, 66, 8469-8477.

(20) Liu, Z.; Chen, Z.; Guo, H.; He, D.; Zhao, H.; Wang, Z.; Zhang, W.; Liao, L.; Zhang, C.; Ni, L. The modulatory effect of infusions of green tea, oolong tea, and black tea on gut microbiota in high-fatinduced obese mice. Food Funct. 2016, 7, 4869-4879.
(21) Gu, F.; Borewicz, K.; Richter, B.; van der Zaal, P. H.; Smidt, H.; Buwalda, P. L.; Schols, H. A. In Vitro Fermentation Behavior of Isomalto/Malto-Polysaccharides Using Human Fecal Inoculum Indicates Prebiotic Potential. Mol. Nutr. Food Res. 2018, 62, No. e1800232.

(22) Ramasamy, U. S.; Venema, K.; Schols, H. A.; Gruppen, H. Effect of soluble and insoluble fibers within the in vitro fermentation of chicory root pulp by human gut bacteria. J. Agric. Food Chem. 2014, 62, 6794-6802.

(23) Magoč, T.; Salzberg, S. L. FLASH: fast length adjustment of short reads to improve genome assemblies. Bioinformatics 2011, 27, 2957-2963.

(24) Caporaso, J. G.; Kuczynski, J.; Stombaugh, J.; Bittinger, K.; Bushman, F. D.; Costello, E. K.; Fierer, N.; Peña, A. G.; Goodrich, J. K.; Gordon, J. I.; Huttley, G. A.; Kelley, S. T.; Knights, D.; Koenig, J. E.; Ley, R. E.; Lozupone, C. A.; McDonald, D.; Muegge, B. D.; Pirrung, M.; Reeder, J.; Sevinsky, J. R.; Turnbaugh, P. J.; Walters, W. A.; Widmann, J.; Yatsunenko, T.; Zaneveld, J.; Knight, R. QIIME allows analysis of high-throughput community sequencing data. Nat. Methods 2010, 7, 335-336.

(25) Edgar, R. C. UPARSE: highly accurate OTU sequences from microbial amplicon reads. Nat. Methods 2013, 10, 996-998.

(26) Quast, C.; Pruesse, E.; Yilmaz, P.; Gerken, J.; Schweer, T.; Yarza, P.; Peplies, J.; Glóckner, F. O. The SILVA ribosomal RNA gene database project: improved data processing and web-based tools. Nucleic Acids Res. 2012, 41, D590-D596.

(27) Segata, N.; Izard, J.; Waldron, L.; Gevers, D.; Miropolsky, L.; Garrett, W. S.; Huttenhower, C. Metagenomic biomarker discovery and explanation. Genome Biol. 2011, 12, R60.

(28) Ganguly, S.; G, T. K.; Mantha, S.; Panda, K. Simultaneous determination of black tea-derived catechins and theaflavins in tissues of tea consuming animals using ultra-performance liquid-chromatography tandem mass spectrometry. PLoS One 2016, 11, No. e0163498.

(29) Miketova, P.; Schram, K. H.; Whitney, J.; Li, M.; Huang, R.; Kerns, E.; Valcic, S.; Timmermann, B. N.; Rourick, R.; Klohr, S. Tandem mass spectrometry studies of green tea catechins. Identification of three minor components in the polyphenolic extract of green tea. J. Mass Spectrom. 2000, 35, 860-869.

(30) Yassin, G. H.; Koek, J. H.; Jayaraman, S.; Kuhnert, N. Identification of novel homologous series of polyhydroxylated theasinensins and theanaphthoquinones in the SII fraction of black tea thearubigins using ESI/HPLC tandem mass spectrometry. J. Agric. Food Chem. 2014, 62, 9848-9859.

(31) Tanaka, T.; Betsumiya, Y.; Mine, C.; Kouno, I. Theanaphthoquinone, a novel pigment oxidatively derived from theaflavin during tea-fermentation. Chem. Commun. 2000, 15, 1365-1366.

(32) Verloop, A. J. W.; Vincken, J.-P.; Gruppen, H. A tandem mass spectrometry method based on selected ions detects low-abundance phenolics in black tea-theatridimensins as products of the oxidative cascade. Rapid Commun. Mass Spectrom. 2016, 30, 1797-1805.

(33) Hong, Y.; Guo, J.; Sun, G. Characteristics and phylogenetic analysis of the facultative anaerobic dissimilatory azoreducing bacteria from activated sludge. Int. Biodeterior. Biodegrad. 2008, 61, 313-318.

(34) Li, M.; Gu, J.-D. Molecular evidence of the existence of anaerobic ammonia oxidation bacteria in the gut of polychaete (Neanthes glandicincta). Appl. Environ. Biotechnol. 2016, 1, 19-29.

(35) Stieb, M.; Schink, B. Anaerobic oxidation of fatty acids by Clostridium bryantii sp. nov., a sporeforming, obligately syntrophic bacterium. Arch. Microbiol. 1985, 140, 387-390.

(36) Gross, G.; Jacobs, D. M.; Peters, S.; Possemiers, S.; van Duynhoven, J.; Vaughan, E. E.; Van de Wiele, T. In vitro bioconversion of polyphenols from black tea and red wine/grape juice by human intestinal microbiota displays strong interindividual variability. J. Agric. Food Chem. 2010, 58, 10236-10246.

(37) Jandhyala, S. M.; Talukdar, R.; Subramanyam, C.; Vuyyuru, H.; Sasikala, M.; Nageshwar Reddy, D. Role of the normal gut microbiota. World J. Gastroenterol. 2015, 21, 8787.

(38) Wang, J.; Tang, L.; Zhou, H.; Zhou, J.; Glenn, T. C.; Shen, C.L.; Wang, J.-S. Long-term treatment with green tea polyphenols 
modifies the gut microbiome of female sprague-dawley rats. J. Nutr. Biochem. 2018, 56, 55-64.

(39) Tan, H.; Zhai, Q.; Chen, W. Investigations of Bacteroides spp. towards next-generation probiotics. Food Res. Int. 2019, 116, 637644.

(40) Taylor, P. W.; Hamilton-Miller, J.-M. T.; Stapleton, P. D. Antimicrobial properties of green tea catechins. Food Sci. Tech. Bull. 2005, 2, 71-81.

(41) Lee, H. C.; Jenner, A. M.; Low, C. S.; Lee, Y. K. Effect of tea phenolics and their aromatic fecal bacterial metabolites on intestinal microbiota. Res. Microbiol. 2006, 157, 876-884.

(42) Kerimi, A.; Kraut, N. U.; da Encarnacao, J. A.; Williamson, G. The gut microbiome drives inter- and intra-individual differences in metabolism of bioactive small molecules. Sci. Rep. 2020, 10, 19590.

(43) Eker, M. E.; Aaby, K.; Budic-Leto, I.; Rimac Brnčić, S.; El, S. N.; Karakaya, S.; Simsek, S.; Manach, C.; Wiczkowski, W.; de PascualTeresa, S. A review of factors affecting anthocyanin bioavailability: Possible implications for the inter-individual variability. Foods 2020, 9, 2. 